Rorschach Comprehensive System Variables and Assessment for Psychodynamic Psychotherapy 



\title{
Rorschach Comprehensive System Variables and \\ Assessment for Psychodynamic Psychotherapy
}

\author{
Marianne Nygren
}

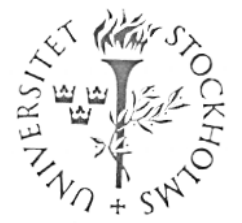

Department of Psychology

Stockholm University

2005 


\title{
Doctoral dissertation 2005 \\ Department of Psychology \\ University of Stockholm \\ SE-106 91 Stockholm, Sweden
}

\begin{abstract}
Rationally selected Comprehensive System (CS; Exner, 1991, 1993, 2003) Rorschach variables and suitability for psychodynamic psychotherapy were investigated in four studies with different designs. The participants comprised three clinical samples $(n=52,25$, and 69) of patients applying for and/or selected for dynamic psychotherapy. With few exceptions the CS variables were the same in the studies, and in all the studies the Rorschach was independent of clinical ratings/assessments and/or selection of patients for treatment. The first study dealt with the relation between 17 rationally selected CS variables and suitability for psychotherapy as represented by ratings of Dynamic Capacity and Ego Strength and by selection of therapy applicants for psychotherapy. The second and third studies concerned CS score differences between groups of patients differing in suitability for dynamic psychotherapy. In the second study, differences for CS variables were predicted for three groups of dynamic psychotherapy patients with mixed diagnoses. In the third study, CS score differences between two groups, differing in suitability but similar in comprising personality disordered patients, were predicted and tested. In the fourth study, correlations were predicted between 18 pre-therapy CS variables and patient post-therapy satisfaction with therapy and also with therapist post-therapy ratings of their patients' suitability for dynamic therapy.

For the four studies taken together, positive results were obtained for EA, FC, the D Score, Blends, Zf, F\%, MQo, and AG. The results for m, SumT, WSUM6, X-\%, S, and FrrF were negative, whereas the results for YFY, FD, COP, and PER were too weak to permit interpretation.
\end{abstract}

The main limitation of the research in this thesis is the lack of psychometric data concerning the clinical ratings whereas the strength is the ecological validity. The results are positive for some of the CS variables selected, mainly concerning control and cognition but also touching upon emotional integration and interpersonal forcefulness. The usefulness of the CS in psychotherapy assessment may be enhanced if only those CS variables that are found valid for the purpose are used and general personality descriptions are avoided.

Key words: Rorschach, Comprehensive System, psychotherapy assessment, psychotherapy suitability.

\section{(C) 2005 Marianne Nygren}

ISBN 91-628-6407-6

Grafisk form Inge Skog, www.akademitjanst.se.

Printed with concern for the environment on chlorine-free paper

by Akademitryck AB, Edsbruk (www.akademitryck.se) 2005. 


\section{Acknowledgements}

First of all I thank my supervisor Professor Carl-Otto Jonsson, this thesis owes much to him. He has generously given his time and support and has shown genuine interest. Without his experience and patience this work would not have been possible. I know I am going to miss the kind of contact we have had during these years. I am grateful to Professor Rolf Sandell, who been generous with his time and has offered advice and support that has been of invaluable and crucial importance.

The research in the first study was carried out at a psychotherapy training unit in Stockholm. This work, that laid the ground for the thesis, would not have been possible without the commitment of Karin Gyllensköld and Per Stenfelt. I am also grateful to the other members of staff at the training unit and last but not least this work was totally dependent on the ratings carried out by the students at the unit and the participation of the therapy applicants.

In two articles I have used data from the Stockholm Comparative Psychotherapy Study, and I thank Anna Maria Carlsson, head of this project, for giving me the opportunity to take part in the project and for support, stimulating discussions and good companionship. I am indebted to the patients in the project who accepted to participate in the research.

Anna Maria Carlsson, Berit Bihlar, Anita Enger, Thomas Lindgren, and Kristina Nordström have contributed with reliability scoring of Rorschach protocols. I specially appreciate Harald Janson's generous sharing of the statistical software Rorschach Research Utilities.

Carl-Otto Jonsson's wife Boel Jonsson is an experienced psychotherapist and psychoanalyst and I have very much appreciated her interest both during the ups and downs this work.

I am grateful to Lena Lilja, head of the County Council unit where I work, for her support. I also thank Steve Wicks for patiently checking the English in different versions of the manuscripts.

I want to express my deep gratitude to my dear husband of so many years, Arne, who has never been impatient with my time-consuming commitments outside our private life. Instead, he has involved himself as discussion partner and proof-reader for some of the manuscripts. I also want to express my great appreciation of the interest, support and help from our two sons Martin and Ola during this long work. 
The thesis is based on the following papers:

1. Nygren, M. (2004). Rorschach Comprehensive System variables in relation to assessing Dynamic Capacity and Ego Strength for psychodynamic psychotherapy. Journal of Personality Assessment, 83, 276-291.

2. Nygren, M. (2004). Differences in Comprehensive System Rorschach variables between groups differing in therapy suitability. Rorschachiana, 26, 110-146.

3. Nygren, M. (submitted). Was the patient satisfied? Was the patient suitable? Pre-therapy Comprehensive System variables in relation to post-therapy Dynamic Capacity and Patient Satisfaction. A clinical study. 


\section{Table of Contents}

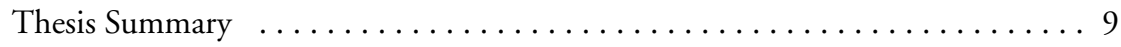

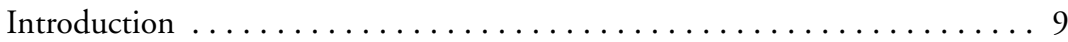

The Rorschach controversy $\ldots \ldots \ldots \ldots \ldots \ldots \ldots \ldots \ldots \ldots \ldots \ldots$

Research concerning the CS and psychotherapy $\ldots \ldots \ldots \ldots \ldots \ldots 12$

Focus and general outline of the present studies $\ldots \ldots \ldots \ldots \ldots \ldots 16$

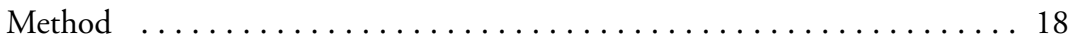

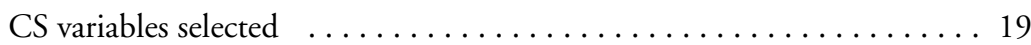

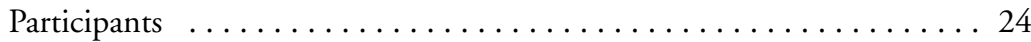

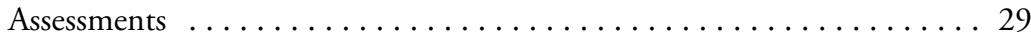

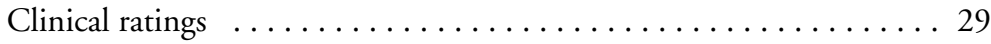

Rorschach administration and scoring $\ldots \ldots \ldots \ldots \ldots \ldots \ldots \ldots \ldots$

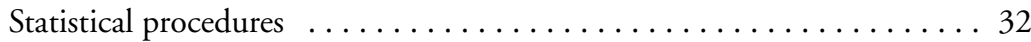

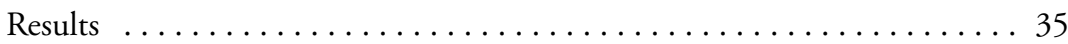

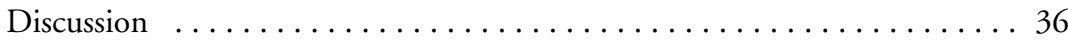

Pattern of results and agreement with earlier research $\ldots \ldots \ldots \ldots \ldots 37$

Interdependence of supported CS variables $\ldots \ldots \ldots \ldots \ldots \ldots \ldots \ldots$

Comments on some single CS variables $\ldots \ldots \ldots \ldots \ldots \ldots \ldots \ldots 40$

Structural and qualitative analysis of the Rorschach $\ldots \ldots \ldots \ldots \ldots . \ldots 1$

Implications for clinical psychotherapy assessment $\ldots \ldots \ldots \ldots \ldots \ldots 42$

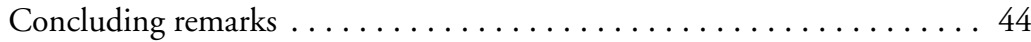

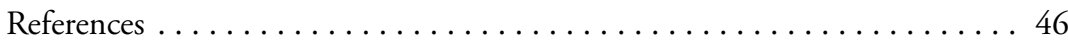

Paper I (Study 1)

Rorschach Comprehensive System variables in relation to assessing

Dynamic Capacity and Ego Strength for psychodynamic psychotherapy . 55

Paper II (Study 2 and 3):

Differences in Comprehensive System Rorschach variables between groups differing in therapy suitability $\ldots \ldots \ldots \ldots \ldots \ldots \ldots \ldots, 91$

Paper III (Study 4)

Was the patient satisfied? Was the patient suitable? Pre-therapy

Rorschach Comprehensive System variables in relation to post-therapy rated Patient Satisfaction and Dynamic Capacity. A clinical study . . . . 127 



\section{Rorschach Comprehensive System variables and Assessment for Psychodynamic Psychotherapy}

\section{INTRODUCTION}

The Test stimuli of the Rorschach method comprise ten cards with symmetrical inkblots printed on them. The inkblots on the first seven cards are in black and white with the exceptions of card II and III, which have a few red details. The last three cards are printed in pastel colours. The method dates from 1921 when Hermann Rorschach first published his work about inkblots used as stimulus figures in the monograph 'Psychodiagnostik', which was later (1942) also published by the Huber Verlag. Rorschach thought that inkblots could serve as stimulus figures and be a diagnostic help in the differentiation of schizophrenia. He developed a scoring system based on empirical grounds, and when he unexpectedly died 1922 he was planning more research on what he called the 'form interpretation test'.

As Hermann Rorschach died young and was unable to fulfil his research on the 'form interpretation test', various persons became influential on the development of the method. Among the most well known were Samuel Beck, Bruno Klopfer, Marguerite Hertz, Zygmunt Piotrowski, and David Rapaport. Different views on how to develop Hermann Rorschach's method led to the development of different Rorschach systems. The systems differed in many respects; they had different instructions, different criteria for the application of Rorschach's original symbols, different symbols for achromatic and shading responses (not included in Rorschach's work), and different ways of interpreting the Rorschach.

The Rorschach Research Foundation (Rorschach Workshops) was established in 1968 by John Exner Jr. Questionnaires to clinicians and the creation of a data pool that permitted a study of the differences of the five main Rorschach systems (the Klopfer, the Beck, the Rapaport, the Piotrowski, and the Hertz systems) were important undertakings for the foundation. This work led to the 
The CS and assessment for dynamic psychotherapy

decision to 'integrate the features of all systems for which empirically defensible data existed or could be established' (Exner, 1993 p. 23). Exner and his coworkers systemised Rorschach administration and scoring procedures, and made a normative work based on a large archive of structural aspects of Rorschach protocols from non-patients and various psychiatric patient groups. In 1974 'The Rorschach: A Comprehensive System' was published (Exner, 1974), and this volume has been followed by three more editions.

\section{The Rorschach controversy}

The extensive contribution by Exner and his co-workers has evoked a great interest and positive reactions, but since the mid nineties harsh criticism has also been levelled at the Comprehensive System (CS). Central issues have been the empirical foundation of the CS, the psychiatric diagnostic validity of the CS, and the CS norms. Reliability questions, incremental validity and clinical utility, quality of meta-analytical studies supporting the Rorschach, and sometimes single CS variables have also been intensely debated.

The Rorschach Workshops, which constitute the main empirical foundation for the CS, have been criticised for relying too heavily on unpublished studies of questionable value (Garb, Wood, Lilienfeld, \& Nezworski, 2002; Garb, Wood, Nezworski, Grove, \& Stejskal, 2001; Hunsley \& Bailey, 2001; Wood, Nezworski, \& Stejskal, 1996a, 1996b).

The CS has been criticised for deficiencies in psychiatric diagnostic validity (Carlsson, Kula, \& Laurent, 1997; Garb et al., 2001; Garb et al., 2002; Garfield, 2000; Lilienfeld, Wood, \& Garb, 2000; Wood, Lilienfeld, Garb, \& Nezworski, 2000a, 2000b).

The norms of the CS have been seriously questioned. In a study of nonpatient adults, Shaffer and Erdberg (1999) obtained results for many CS variables that differed from the data in Exner's Comprehensive System Workbook (1995). These findings have resulted in a series of articles debating the CS norms and the risk of false positive identifications of psychopathology in clinical and forensic settings (Aronow, 2001; Exner, 2001; Exner, 2002; Garb, 2003; Garb, et al., 2002; Hibbard, 2003; Hunsley \& Di Giulio, 2001; Lilienfeld et al., 2000; Meyer, 2001; Wood, Nezworski, Garb, \& Lilienfeld, 2001a, 2001b). However, Exner has reported that he is now working on calculating CS norms for a new non-patient sample, using essentially the same design and exclusion criteria as 
in the original project. Results for the first 175 participants are reported to be quite similar to those in the original project (Exner, 2002).

Proponents and critics of the CS have also debated the temporal consistency and the interrater reliability of the CS (Acklin, McDowell, Verschell, \& Chan, 2000; Costello, 1999; Ganellen, 2001; Garb et al., 2001; Hibbard, 2003; Hunsley \& Bailey, 1999; Lilienfeld et al., 2000; McDowell \& Acklin, 1996; Meyer, 1997a, 1997b; Meyer et al., 2002; Viglione, 1999; Viglione \& Hilsenroth, 2001; Weiner, 2001; Wood, Lilienfeld, Nezworski, \& Garb, 2001; Wood, Nezworski, \& Stejskal, 1996a, 1996b; Wood, Nezworski, \& Stejskal, 1997). In research, the reliability of the CS variables is now mostly calculated by using the intraclass correlation coefficient, ICC, as recommended by among others Acklin et al. (2000).

The incremental validity and clinical utility of the CS has been seriously questioned (Garb et al., 2001; Hunsley \& Bailey, 1999, 2001), while other authors have presented support for the incremental validity and utility of the CS (Blais, Hilsenroth, Castlebury, Fowler, \& Baity, 2001; Meyer, 2000; Viglione, 1999)

An issue that has been central in the critique of the validity of the CS concerns the meta-analytic studies undertaken primarily to obtain information about the general validity of the Rorschach. There are meta-analytic studies (Atkinson, Quarrington, Alp, \& Cyr, 1986; Bornstein, 1999; Hiller, Rosenthal, Bornstein, Berry, \& Brunell-Neulib, 1999; Meyer \& Handler, 1997; Parker, Hanson, \& Hunsley, 1988), which are regarded as supporting the validity of the Rorschach. However, these studies have been criticised as being methodologically defective, and several researchers have engaged themselves in a controversy concerning the value of the analyses (Garb et al., 2001; Garb, Florio, \& Grove, 1998, 1999; Hunsley \& Bailey, 1999; Lilienfeld et al., 2000; Meyer \& Archer, 2001; Parker, Hunsley, \& Hanson, 1999; Rosenthal, Hiller, Bornstein, Berry, \& Brunell-Neulieb, 2001; Viglione \& Hilsenroth, 2001; Weiner, 2001).

The debate has also sometimes focused on single CS variables. Kleiger (1992) formulated a conceptual critique concerning the interpretation of EA (Experience Actual) in the CS interpreted to indicate accessibility to psychological resources, and the D Score (difference between EA and es, experienced stimulation) in the CS interpreted to indicate degree of experienced psychological stress. Nezworski and Wood (1995) and Wood et al. (1996a, 1996b) criticised the CS interpretations of the Egocentricity Index, and of the Reflection responses as indicating narcissistic-like features. They also criticised the 
CS interpretations of the D Score, of the Depression Index, of the Suicide Constellation, of $\mathrm{Hx}$ (Human Experience score), in the CS indicating an intellectualising way of dealing with self-image, and of Food responses as indicating dependency problems. Hilsenroth, Fowler, Padawer, and Handler (1997), however, obtained results supporting the value of reflection responses, pair responses, the Egocentricity Index, and PER (personalised responses), interpreted to indicate intellectual authoritarianism, as CS variables differentiating persons with narcissistic personality disorder from non-patients and from patients diagnosed as Cluster $\mathrm{A}, \mathrm{B}$, or $\mathrm{C}$ personality disorders according to DSMIV (American Psychiatric Association, 1994).

To sum up, the critique has questioned the scientific grounds and the norms of the CS and has commented upon the risk for over-pathologisation depending on the problem with the norms; the risk for serious consequences of this in forensic and clinical assessments has been emphasised. The reliability and the psychiatric diagnostic validity of the CS have also been questioned, and metaanalytical studies supporting the value of the CS have been criticised for being flawed.

The studies in this thesis do not address the CS norms, the CS validity for psychiatric diagnoses, or the use of the CS in connection with forensic decisions. The research in the present thesis concerns selected CS variables and assessment for psychodynamic psychotherapy, and the focus is not on psychiatric pathology but on assets and liabilities of relevance for the possibility to engage in psychodynamic psychotherapy. Garb et al. (2002) recommended that psychologists working in the fields of applied psychological investigation should only use CS scores that have been researched and found valid and reliable for the question under investigation. This is a recommendation in line with the reasoning by Weiner $(1996,2001)$ concerning the multidimensionality of the Rorschach and the need to calculate the validity of different parts of the method for various purposes. This is the point of departure for the present studies; the aim was to contribute to the study of the value of selected CS variables in assessment for psychodynamic psychotherapy.

\section{Research concerning the CS and dynamic psychotherapy}

Over the past twenty years, research focusing on ego functioning related to suitability for psychodynamic psychotherapy has yielded positive findings. Re- 
searchers have shown considerable interest in psychological mindedness, quality of relations, and motivation as possible predictors of completion and outcome of psychodynamic psychotherapy and as predictors of the patient-therapist relationship.

Psychological mindedness has been studied by several researchers and received positive results as related to number of psychotherapy sessions attended, outcome of dynamic psychotherapy, and staying in therapy (Conte et al., 1990; Conte, Buckley, Picard, \& Karasu, 1995; Høglend, Engelstad, Sørbye, Heyerdahl, \& Amlo, 1994; McCallum \& Piper, 1990a, 1990b; McCallum, Piper, \& Joyce, 1992; Piper, Joyce, Rosie, \& Azim, 1994; Tasca et al., 1999).

Pre-treatment quality of patients' interpersonal relations is another variable that has interested researchers and that has been studied in combination with psychological mindedness. A clinical implication is that assessment of quality of object relations can yield information that is valuable and relevant for interpretations of transference in psychodynamic psychotherapy (Piper \& Duncan, 1999). Results have indicated that quality of relations is related to outcome of interpretive but not supportive dynamic psychotherapy, to therapeutic alliance and to improvement (Høglend, 1993; Piper et al., 1991; Moras \& Strupp, 1982; Piper, Joyce, Azim, \& Rosie, 1994; Piper, Joyce, McCallum, \& Azim, 1998; Piper, Azim, McCallum, Joyce, \& Ogrodniczuk, 1999).

Motivation has received support as predictor of how an individual will benefit from psychotherapy (Garfield, 1994; Høglend,1996; Høglend, Sørbye, Sørlie, Fossum, \& Engelstad, 1992; Keithly, Samples, \& Strupp, 1980; Orlinsky, Grawe, \& Parks, 1994; Roth \& Fonagy, 1996; Sifneos, 1978).

There is thus a wealth of studies demonstrating that psychological mindedness, quality of object relations and motivation in particular are related to psychotherapy outcome, favourable patient-therapist relationship or completion of therapy. The assessment methods employed have mostly been ratings by interviewers or observers, the Psychological Mindedness Assessment Procedure ([PMAP] McCallum \& Piper, 1990a), and patients' self-ratings.

However, the evidence concerning the value of CS Rorschach variables in assessment for psychodynamic psychotherapy is more limited. Alpher, Perfetto, Henry, and Strupp (1990) conducted a study to investigate suitability for dynamic psychotherapy. Fifteen rationally chosen CS indicators and independent ratings of 'The Capacity for Dynamic Process Scale' (CDPS), focusing on the patient's observable capacity to collaborate therapeutically during a semistructured intake interview, the Interpersonal Assessment Interview, were studied. Organisational activity (Zf), interpreted in the CS to be related to ambi- 
tiousness and motivation, texture shading responses (SumT), indicating capacity for close relations with others, Blends, in the CS indicating capacity to deal with complex emotional stimuli, and inanimate movement responses $(\mathrm{m})$ interpreted to indicate experience of inner tensions, were positively related to the CDPS. Pure form proportion (Lambda), interpreted as related to openness of experience, high values indicating low degree of involvement, was negatively related to the CDPS. Number of Deviant Responses (DR), in the CS interpreted to indicate cognitive slippage, was also positively related to the CDPS, but was interpreted as a possible positive predictor of a patient's potential for engaging in the process of a dynamic psychotherapy 'only given an otherwise intact personality' (Alpher et al., 1990, p. 227).

LaBarbera and Cornsweet (1985) predicted relations between psychological flexibility, as represented by the constructs of instability and perceptual sensitivity, and outcome of dynamically-oriented treatment of child psychiatric inpatients. Instability and perceptual sensitivity were measured by rationally selected CS scores. Children benefiting from treatment demonstrated perceptual sensitivity, as indicated by lower values on Lambda, higher values of Blends, and of organisational effort (Zf and ZSum), and they also showed greater instability as indicated by a greater difference between irritating stimuli (es) and organising resources (EA).

Bihlar and Carlsson $(2000,2001)$ reported two exploratory studies related to the usefulness of the CS for treatment planning in psychodynamic therapies. The first study concerned agreement between psychodynamically oriented therapists' therapy-goal formulations and patients' problems as indicated by the CS. In the second study, Bihlar and Carlsson (2001) compared CS scores in two groups. In the first group, there was a high level of agreement between the therapists' planned goals and the actual goals reported by the therapists after therapy, and in the second group, the agreement between planned and actual goals was low. Selected CS scores and combinations of CS scores differed between the two groups. Bihlar and Carlsson contended that for some patients the standard clinical interview might not be an adequate instrument for making assessments for dynamic therapy, that additional instruments might yield valuable information about the patient, and that the Rorschach might provide information difficult to obtain in pre-therapy interviews.

Weiner and Exner (1991) predicted 27 CS indices of adjustment difficulty to diminish over time among patients in psychotherapy. The indices were chosen so as to be related to personality characteristics associated with desirable goals in long-term psychodynamic therapy. Two demographically similar 
groups were studied, 88 patients in long-term dynamic therapy and 88 patients in short-term psychotherapy. The Rorschach was administered at the beginning of therapy, after one year, after 2.5 years, and after four years. The results showed significant changes in the predicted direction for all 27 indices except for Depression index (DEPI > 5) and reflection responses ( $\mathrm{FrF}>0)$.

Using an exploratory test-retest design, Exner and Andronikof-Sanglade (1992) studied one group of patients receiving brief ( $M=14.2$ sessions), and one group receiving short-term psychotherapy ( $M=47$ sessions). Demographically the two groups were similar. Rorschach protocols and a self-report measure, Katz Adjustment Scales-Form, were administered before entering therapy, at termination of therapy, and 10 to 12 months after termination. According to the Katz Adjustment Scales-Form, both groups reported fewer symptoms and better satisfaction with free-time activities, both at termination and at 10 to 12 months after termination. Twenty-seven structural Rorschach indexes were studied. Twenty-three of them were identical with those studied by Weiner and Exner (1991). Significant positive changes in 21 Rorschach indexes were obtained in the short-term therapy group at the second retest, whereas only seven indexes showed significant changes in the brief therapy group. The authors concluded that this study, in accordance with the one by Weiner and Exner (1991), gave indirect support to the validity of some structural variables in the Rorschach.

An index of Ego Strength derived from six components of the Rorschach test, the Rorschach Prognostic Rating Scale (RPRS) (Klopfer, Ainsworth, Klopfer, \& Holt, 1954) has been used with some positive results concerning psychotherapy outcome (Garfield, 1994). Meta-analytic studies of the RPRS (Meyer, 2000; Meyer and Handler, 1997, 2000) have given support for the RPRS as a valid scale for prediction of treatment outcome and of substantial utility in selection of patients for psychotherapy. The RPRS was intended to be a measure of overt but also of tacit ego resources that could be mobilized during therapy, thus reflecting therapeutic promise (Meyer \& Handler, 1997). However, the RPRS is a complicated scale based on scoring of movement responses, colour responses, shading responses, and form quality according to the Klopfer Rorschach tradition. Although the RPRS is partly derived from scores similar to those in the CS, it was not included in the CS. Thus, research results concerning the RPRS cannot be regarded as direct support for the CS. Moreover, it is important to search for Rorschach indicators for psychotherapy that are not too complicated to score and use clinically. In this thesis, separate Rorschach variables according to the CS not combined into an index or a scale were studied. 
The present research is most akin to the study by Alpher et al. (1990). However, although Alpher et al. made a rational selection of CS variables, the conceptual ground for the selection was not made clear. The study by LaBarbera and Cornsweet (1985) focused upon treatment of child psychiatric patients and not directly on psychodynamic psychotherapy. The promising results in the studies by Weiner and Exner (1991), and Exner and Andronikof-Sanglade (1992) are limited because these studies did not contain correlations with other assessment methods that could have contributed to the interpretation of the CS variables. As in the present studies, Bihlar and Carlsson $(2000,2001)$ focused on the possible value of the CS as a complementary instrument to the clinical interview in psychotherapy assessment. However, these studies concerned therapists' assessments as represented by goal formulations, and were of a more exploratory character than this research.

\section{Focus and general outline of the present studies}

Several researchers (Atkinson, Quarrington, Alp, \& Cyr, 1986; Kleiger, 1992; Viglione, 1997; Weiner, 1996, 2000b) following a common rule in research, recommend that predictions in Rorschach validation research should be conceptually based on some rationale grounded in theory or in earlier research. The studies in this thesis were conducted in accordance with these recommendations.

The focus of the research in this thesis was on rationally selected CS structural variables that according to their interpretations in the CS should be related to ego functioning variables relevant for psychodynamic psychotherapy. The ratings of ego functioning variables in the present thesis are more akin to the everyday clinical assessments performed by psychotherapists before making decisions concerning psychotherapy than to more advanced assessment scales like the CDPS, used by Alpher et al. (1990).

The present research comprises four studies with different designs, where the results should be expected to be in the same direction. The results in one study did not influence the predictions in the others. For the convenience of the reader, in the following the number of each study is accompanied by a shortened variant of its title.

Study 1 (Paper I), Pre-treatment CS, pre-treatment Dynamic Capacity and Ego Strength. The aim of this study was to investigate the relation between CS 
variables and clinical ratings of Dynamic Capacity (suitability for dynamic psychotherapy) and Ego Strength. Correlations between CS variables and clinical ratings of Dynamic Capacity and Ego Strength were predicted and calculated. CS variables for the applicants selected for psychotherapy and for the applicants not selected, were also compared using independent samples t-test.

Study 2 (Paper II), CS and three groups differing in therapy suitability, focused on predicted differences in CS variables between three demographically similar groups of patients in psychodynamic psychotherapy that differed in therapy suitability. Two groups of patients assumed to have higher levels of suitability for dynamic psychotherapy were contrasted with a third group, assumed to have a lower level of therapy suitability. The third group comprised therapy patients with at least one diagnosis of personality disorder according to DSMIII-R (APA, 1987) who had proven difficult to treat in the framework of the County Council psychiatry service. However, the patients in the third group were still deemed possible to help in an individually designed outpatient program with dynamic psychotherapy.

Study 3 (Paper II), CS and two personality disorder groups with different therapy suitability, predicted differences between two groups of psychotherapy patients assumed to differ in suitability for psychodynamic psychotherapy. The two groups were demographically similar groups of patients, all with personality disorder according to DSM-III-R (APA, 1987) or DSM-IV (APA, 1994).

Study 4 (Paper III), on pre-treatment CS, post-treatment Dynamic Capacity and Patient Satisfaction, focused on the predicted correlations between pretreatment $\mathrm{CS}$ variables and post-treatment rated suitability for psychotherapy, and between pre-treatment CS variables and post-therapy patient evaluation of therapy. Moreover, CS variable comparisons between patients not satisfied and patients medium to highly satisfied with their therapy were carried out using independent samples t-test. 
The CS and assessment for dynamic psychotherapy

\section{METHOD}

After the description of the selected CS variables, the studies will be described in some detail, one after the other, under the headings 'Participants', 'Measures', and 'Statistical procedures'. For the sake of clarity, a table presenting the studies in the respective aspect introduces each of these sections.

However, first a brief orientation is given to help readers who are not familiar with CS. During the administration of the Rorschach all responses are recorded verbatim. The responses are then coded/scored in several aspects. The CS scoring of the responses constitutes the basis for the Structural Summary, the database for the interpretations, comprising the composite of the code frequencies plus a number of ratios, and percentages. However, in the present research most of the CS variables selected have a low degree of complexity.

Location scores indicate what part(s) of the blot that the subject used. There are three codes for location; W (whole blot), D (common detail), and Dd (uncommon detail). If the white space is used the symbol $S$ is added to the location code. A symbol for Developmental Quality is added to the Location score; there are four levels ranging from vague responses to synthesized responses.

Determinants are then coded to denote which features of the blot contributed to the answer formulated. The aspects coded are pure form, chromatic colour, achromatic colour, three kinds of shading (texture, vista, and diffuse shading), and three kinds of movement (human, animal, and inanimate). Moreover, there are determinant codes for Reflection responses and for Form Dimension (responses including three dimensions based exclusively on form and not on the shading qualities of the blot). The appropriateness of the form used is added to the determinant symbol. There are four levels of form appropriateness ranging from superior/overelaborated to distorted unrealistic use of the form in creating the response.

The contents of the response are coded with symbols that are mainly abbreviations, such as $\mathrm{H}$ for human, $\mathrm{A}$ for animal, and Fd for food etc. If the response is popular this is denoted by the code $\mathrm{P}$.

Organizational activity is scored for responses involving the whole blot, and for responses that involve integrating or organizing the parts of the blot in a complex manner. A numerical value, a Z-score is assigned to the answer to denote degree of organizational activity.

Finally, Special Scores are coded. There are mainly two kinds of Special Scores, one concerns cognitive slippage and occurrence of disordered thinking and the other concerns sets in self and interpersonal perception. 


\section{CS variables selected}

In all, 19 CS variables were selected in the present research. However, three of these were not included in all studies. SumT was not included in Study 2, the D Score was not included until Study 3, and the Coping Deficit Index was only included in one study, Study 3 . These exceptions will be commented on in the presentation of the CS variables below. A detailed description of the CS variables and the rationale for the direction of the predictions are included in Study 1, Pre-treatment CS, pre-treatment Dynamic Capacity and Ego Strength. However, a summary of the variables selected and the direction of the predictions are presented below.

\section{CS variables concerning control and stress tolerance}

Experience actual, EA, is calculated as the sum of M (human movement answers) and WSUMC (weighted sum colour answers). It is interpreted as indicating available resources and capacity to implement deliberate coping strategies, and as related to but not the same as ego strength. In the present research, EA was included as a probable positive indicator for psychodynamic psychotherapy.

The form dominant colour response, FC, also one of the scores included in the EA, is interpreted to be related to affective experiences that are cognitively directed and well controlled (Exner, 1993). Although a dominance of FC responses can be a sign of very, perhaps too, strong control of emotions, FC was regarded as indicating mainly positive capacities in this thesis. $\mathrm{FC}$ was predicted to be a positive indicator for dynamic psychotherapy, positively related both to therapy suitability and ego strength.

Diffuse shading responses (FY, YF, and Y) are interpreted as indicating an experience of stress-related psychological helplessness and inability to influence one's situation (Exner, 1991, 1993). It seems probable that form dominance in diffuse shading answers is qualitatively different from non-form dominance, and related to better ego functioning than non-form dominance (Kleiger, 1992, 1997; Meyer, 1992). To feel unable to influence one's situation was regarded as hindering motivation for psychotherapeutic co-operation, and in the predictions in this thesis non-form dominant diffuse shading CS responses, YFY, were considered as a possible negative indicator for dynamic psychotherapy and negatively related to ego strength.

Inanimate movement responses, $\mathbf{m}$, are interpreted as indicating inner ten- 
sions and a feeling of forces being outside the control of the subject. Often the tension is interpreted to be of situational character (Exner, 1991, 1993). In the study by Alpher et al. (1990), m related positively to the Capacity for Dynamic Process Scale, and Weiner (1998) contended that lack of $\mathrm{m}$ often indicates indifference and deficient concern, whereas elevations of $m$ indicate concern about being exposed to forces outside one's control. Other authors have conceptualised $\mathrm{m}$ as positively related to suitability for psychodynamic psychotherapy. Lerner (1991) regarded $\mathrm{m}$ as indicating repressed material coming into awareness in the form of subjectively experienced tensions, and thereby giving opportunity for contact around the patient's feelings of distress. Klopfer et al. (1954) saw $\mathrm{m}$ as indicating an awareness of forces out of control and contended that $\mathrm{m}$ indicates strength because the individual does not use dissociation and disintegration. Inanimate movement is also one of the scores in the RPRS. In the present research, $\mathrm{m}$ was predicted to be positively related to suitability for dynamic psychotherapy.

Sum T, number of texture responses, is interpreted to be related to a capacity for close relations with other persons. Both an absence and an elevation of Sum $T$ can indicate liabilities related to dependency. Sum $T=0$ is interpreted as indicating a tendency to be guarded and/or distant in interpersonal contacts, while an elevation of SumT $(\mathrm{T}>1)$ can indicate stronger than usual needs to be dependent on others and a feeling of emotional deprivation and interpersonal neediness (Exner, 1993; Weiner, 1998). The inclusion of SumT in the present research and the formulations of the predictions were motivated mainly by the positive result received by Alpher et al. (1990). However, it was difficult to formulate predictions for the groups compared in Study 2, where one group of therapy patients assumed to be less suitable for dynamic psychotherapy was contrasted with two groups assumed to be more suitable. In the group with the lowest suitability, the patients had dependency problems of quite different kinds, some being dependent on others and some being much distanced in relations. Therefore, Sum T was excluded in Study 2, but in Study 3 Sum T=1, indicating a good capacity for close relations, was predicted to be less frequent in the group of personality disorder patients more difficult to treat. Thus, Sum T was included as a possible positive indicator for dynamic psychotherapy in three of the four studies in the present thesis.

The D Score is calculated from the raw score EA-es (experienced stimulation), and is interpreted to indicate ability to maintain control under stress and demand. D Score values exceeding zero indicate greater capacity for control and tolerance for anxiety, while values under -1 indicate a risk for a continu- 
ous state of overload and disorganization in demanding situations in life (Exner, 1993). The idea to include the D Score arose when formulating the predictions of Study 3, CS and two personality disorder groups with different therapy suitability. One of the two groups with personality disorders comprised patients that had difficulties to control themselves and problems with acting out, and $\mathrm{D}$ Score $<0$ was predicted to be more frequent in this group. However, positive values (D Score $>0$ ) may indicate difficulties in psychotherapy; patients tend to use their control capacity to avoid the feelings of discomfort involved in and promoting growth or change (Exner 1993; Weiner, 1998). The D Score was kept in Study 4, on pre-treatment CS, post-treatment Dynamic Capacity and Patient Satisfaction, and was predicted to be negatively related to therapy suitability and to patient satisfaction with therapy.

The CDI, the Coping Deficit Index, is interpreted as related to social immaturity and difficulties in coping with the requirements of everyday living, and to being sensitive to depression not marked mainly by dysphoric affect and negative thoughts but rather by pervasive helplessness (Exner, 1991, 1993; Weiner, 1998). However, the CDI was only included in Study 3, CS and two personality disorder groups with different therapy suitability, as indicating a probable diagnostic difference between the two groups. It should therefore be regarded as 'off the record' in the present research.

Blends, which consist of two or more determinants combined in a single response, are mostly interpreted as involving some affective experience (Exner, 1991, 1993). Few Blends may indicate difficulties in situations that are emotionally complex. Although the psychological complexity indicated by high proportions of Blends can be related to difficulties in dealing with affect for persons with limited control resources, Blends often indicate an asset (Exner, 1993). In this thesis, number of Blends was regarded to indicate an asset in psychodynamic psychotherapy and it was predicted to be positively related to therapy suitability and ego strength.

\section{The cognitive triad:}

CS variables focusing on information processing, ideation, \& mediation

Frequency of organisational activity, $\mathbf{Z f}$, is conceptualised as an indicator of willingness to attempt to synthesise the world in a meaningful way. It is also regarded as being related to a person's ambitiousness and motivation (Exner, 1991, 1993). Zf was positively related to the Capacity for Dynamic Process Scale in the Alpher et al. study (1990), and in the LaBarbera and Cornsweet 
study (1985) children classified as improvers had more Zf responses than children classified as decliners. In the present thesis, $\mathrm{Zf}$ was regarded as representing assets important in dynamic psychotherapy and was predicted to be positively related to therapy suitability.

The relative amount of pure form responses, $\mathrm{F} \%$, is interpreted as being related to openness to experience, high values indicating detachment and lack of flexibility (Exner 1991, 1993; Weiner, 1998). In the present research, F\% was used instead of the CS structural summary variable Lambda (L), as it is conceptually and mathematically comparable to L but has better research properties (Meyer, Viglione, \& Exner, 2001). In the Alpher et al. study (1990), L was inversely related to the Capacity for Dynamic Process Scale, and in the LaBarbera and Cornsweet study (1985) L was lower among the children classified as improvers. In this thesis, F\% was considered a negative indicator for psychodynamic psychotherapy indicating lack of flexibility and rigid psychological defences, and predicted to be negatively related to ego strength and therapy suitability.

In the present research, human movement responses with ordinary form quality, MQo, were regarded as a positive indicator for dynamic psychotherapy. Human movement responses are generally interpreted as related to an innerdirected orientation and a capacity for imagination and fantasy (Blatt \& Ford 1994; Exner, 1993; Lerner, 1991). Accurately seen human movement is regarded to indicate empathic capacity, whereas distorted $\mathrm{M}$ responses indicate lack of empathic capacity (Weiner, 1998). Moreover, $\mathrm{M}$ is an important score in the RPRS, where M answers of good fit with the blot indicate an asset and a good prognostic sign (Klopfer et al., 1954).

Weighted sum special scores, WSUM6, are interpreted as indicating bizarre and disturbed thinking, and higher values as a sign of pathological psychological functioning (Exner, 1991, 1993). Percent poor form level, X-\%, is interpreted as indicating distorted perceptual mediation, an important aspect of reality testing capacity. Both WSUM6 and X-\% were predicted to be negatively related to ego strength in this research.

\section{Affective features}

White space responses, $S$, can be interpreted to indicate an asset, a sound selfassertiveness, although higher values $(>2)$ are interpreted as indicating a negativistic and oppositional set toward the environment (Exner, 1991, 1993; Klopfer et al., 1954; Weiner, 1998). In this research, such negativism was as- 
sumed to be related to patients' resistance, which is associated with negative therapeutic outcome (Orlinsky et al., 1994), and $S$ was predicted to be negatively related to suitability for dynamic psychotherapy.

\section{The self \& interpersonal perception clusters}

Reflection responses, $\mathrm{FrrF}>0$, are interpreted in the CS as indicating narcissistic defensive needs. However, elaborations by Exner (1991) and by Weiner (1998) sound more like descriptions of narcissistic personality than of narcissistic defences. As patients with narcissistic personalities are often regarded as difficult to treat with psychotherapy, especially time-limited therapy (Kernberg, 1975; McWilliams, 1994), FrrF was regarded as a probable negative indicator for dynamic psychotherapy in the present studies.

Form dimension, FD, was identified during the development of the CS. It is interpreted as being related to self-inspecting behaviours (Exner, 1991, 1993). Although more than two FD responses can indicate exaggerated involvement with self-examination, self-inspecting capacity is generally preferable in insight psychotherapy and therefore the overall prediction in this thesis was that FD should be positively related to therapy suitability.

Aggressive movement responses, AG, and co-operative movement responses, COP, are regarded to provide information about the sets that persons have concerning interactions with others (Exner, 1991, 1993). In this thesis, a co-operative set was predicted to indicate an asset, and COP was predicted to be positively related to suitability for dynamic psychotherapy. AG indicates an aggressive/forceful set. However, according to Weiner (1998), a high score on AG is not always an indication of maladaptation, AG may indicate more assertiveness than anger. Hilsenroth, Handler, Toman, and Padawer (1995) found that patients terminating psychodynamic psychotherapy prematurely had fewer AG and more COP than those remaining in treatment. Moreover, research concerning the relation between patients' affective responses and therapy outcome indicates that the patient's total affective reactions to therapy (negative and positive reactions) are related to positive therapy outcome (Orlinsky, et al., 1994). The predictions in this thesis were that not only COP but also AG should be positively related to suitability for psychodynamic psychotherapy.

PER, personalised answers are interpreted to indicate defensiveness, in contrast to openness, as characterised by intellectual authoritarianism (Exner, 1991, 1993). Research indicates that patients' openness is associated with posi- 
tive therapy outcome (Orlinsky, et al., 1994), and in accordance with this, PER was predicted to be negatively related with suitability for psychodynamic psychotherapy.

\section{Participants}

The participants came from three different samples of patients applying for or accepted into psychodynamic psychotherapy. Psychotic patients or patients with alcohol or drug abuse were not included in this research. As described later in the method section, a sample or a sub-sample was in some cases used in more than one study. Table 1 and 2 present an overview of the participants.

Table 1. Summary description of the groups in Study 1-4.

\section{Participants}

Study 1 (Paper I) Fifty-two consecutive patients (16 men and 36 women) applying for individual dynamic psychotherapy performed by students on an advanced psychotherapy training program.

Study 2 (Paper II) Three groups ( $n=30,43$, and 25) of psychotherapy patients, one group with a lower level of therapy suitability than the two other groups. The groups were made similar with regard to age, length of education, and $\mathrm{R}^{\mathrm{a}}$.

Study 3 (Paper II) Two groups ( $n=35$ and 25) of psychotherapy patients, all with personality disorders but differing therapy suitability. The groups were made similar with regard to age, length of education, and $\mathrm{R}$. The percentage of men in the groups was $24 \%$ and $37 \%$, and the percentage of women was $63 \%$ and $76 \%$.

Study 4, Patient Forty-eight psychotherapy patients (17 men and 31 women) from the Satisfaction Nygren S-COMPAS 69 participant sample, who had evaluated their (Paper III) $\quad$ therapy.

Study 4, Dynamic Forty-four psychotherapy patients (20 men and 24 women) from the Capacity $(\text { Paper III })^{\mathrm{b}} \quad$ pist ratings of Dynamic Capacity had been obtained.

a $\mathrm{R}=$ number of Rorschach responses.

b The drop-out was 21 patients for Patient Satisfaction and 25 for Dynamic Capacity, the drop out overlap being 14 patients. 
Table 2. Overlap and distribution of participants in Study 1-4

\begin{tabular}{|c|c|c|c|c|}
\hline & $\begin{array}{l}\text { Study 1, } \\
\text { Paper I }\end{array}$ & $\begin{array}{l}\text { Study 2, } \\
\text { Paper II }\end{array}$ & $\begin{array}{l}\text { Study 3, } \\
\text { Paper II }\end{array}$ & $\begin{array}{l}\text { Study 4, } \\
\text { Paper III }\end{array}$ \\
\hline $\begin{array}{l}\text { Sample } 1 . \\
\text { Fifty-two consecutive } \\
\text { patients applying for } \\
\text { psychotherapy in an } \\
\text { advanced training } \\
\text { program. }\end{array}$ & $\begin{array}{l}\text { All appli- } \\
\text { cants in- } \\
\text { cluded } \\
(n=52)\end{array}$ & $\begin{array}{l}\text { Applicants } \\
\text { selected for } \\
\text { therapy } \\
\text { included } \\
(n=30) .\end{array}$ & & \\
\hline $\begin{array}{l}\text { Sample } 2 . \\
69 \text { patients selected } \\
\text { from the S-COMPASS } \\
\text { Rorschach data base }\end{array}$ & & $\begin{array}{l}\text { Sub-sample } \\
\text { from Sample } \\
2(n=43)^{\mathrm{a}} \text {. }\end{array}$ & $\begin{array}{l}\text { Sub-sample } \\
\text { from Sample } \\
2(n=35)^{\mathrm{a}} \text {. }\end{array}$ & $\begin{array}{l}\text { a/ Those patients from } \\
\text { Sample } 2 \text { that had } \\
\text { evaluated their therapy } \\
(n=48)^{\mathrm{b}} \text {. } \\
\text { b/ Those patients from } \\
\text { Sample } 2 \text { where post- } \\
\text { therapy therapist ratings } \\
\text { of Dynamic Capacity } \\
\text { were obtained }(n=44)^{\mathrm{b}} \text {. }\end{array}$ \\
\hline $\begin{array}{l}\text { Sample } 3 . \\
30 \text { consecutive } \\
\text { patients from a } \\
\text { psychotherapy unit }\end{array}$ & & $\begin{array}{l}25 \text { of the } 29 \\
\text { patients } \\
\text { selected. }\end{array}$ & $\begin{array}{l}\text { The same } 25 \\
\text { group as in } \\
\text { Study } 2 .\end{array}$ & \\
\hline
\end{tabular}

a 26 subjects were included in both Study 2 and 3.

b For 37 participants ratings of both Patient Satisfaction and Dynamic Capacity were obtained.

c One subject was not included because the therapy was ongoing when the Rorschach was administered, two patients were not included because they became psychotic and in need of heavy medication, and one patient had more than 20 years of education.

In three of the studies in this thesis, Study 2, 3, and 4, participants came from a sample of 69 patients selected by Nygren from the Stockholm Comparative Psychotherapy Study ([S-COMPAS] Carlsson, Nygren, Clinton, \& Bihlar, 1996) Rorschach database. Therefore, this S-COMPAS sample is presented before the samples of the single studies.

The target group in the S-COMPAS comprised patients who had at least one DSM-IV diagnosis on Axis I or II (APA, 1994), were seeking psychotherapy, and were regarded by psychiatric clinic assessment teams as needing 
psychotherapy. S-COMPAS was a naturalistic longitudinal project. For the Nygren sample ( $n=69)$ it was required that the therapy was psychodynamic and given by a therapist in private practice. Moreover, it was required that the therapy had not yet started when the Rorschach was administered, and that the therapist had made ratings of the patient's ego functioning.

The Rorschach was completely independent of and did not influence the assessments of the receiving therapists in the Nygren S-COMPAS sample. The therapists did not work in the referring clinic and they did not take part in the assessment of patients at the clinic. Neither the results of the Rorschach assessments nor the patients' records in the psychiatric units were available to the therapists.

Study 1 (Paper I), Pre-treatment CS, pre-treatment Dynamic Capacity and Ego Strength. The subjects in Study 1 comprised 52 consecutive applicants, 36 women and 16 men, seeking psychodynamic psychotherapy given by students on an advanced psychotherapy training program. Prior to the psychotherapy assessment interviews, all applicants met a senior psychiatrist, who was formally responsible for the medical/psychiatric aspects of the program. Applicants who were psychotic, alarmingly depressed, or in acute crisis were recommended to seek help elsewhere and did not return for further assessment. Mean age was 35 years, ranging from 20 to 57 years. The mean years of education was 14 , ranging from 9 to 20 .

Study 2 (Paper II), CS and three groups differing in therapy suitability, compared three groups of subjects. The groups were composed to be similar with respect to age, education and number of Rorschach responses (R).

Group 1 comprised those psychotherapy applicants in Study 1, Pre-treatment CS, pre-treatment Dynamic Capacity and Ego Strength, that were selected for psychotherapy. Thirty patients, 8 males (27\%) and 22 females (73\%), were selected for dynamic psychotherapy, mostly with two years' duration and a frequency of two sessions per week. Mean age was 31.4 years, ranging from 22 to 41 years. Mean years of education was 13.7, ranging from 10 to 18 . One subject with 20 years of education was excluded to make the groups as demographically similar as possible.

Group 2 was a sub-sample ( $n=43$ ) from the 69 subjects of the S-COMPAS, and was selected in the following way. First, all patients receiving long-term ( $\geq$ 2 years) dynamic psychotherapy were included. Then, to make groups similar, 10 subjects with less than 10 years education, 11 subjects older than 42 years, and 2 subjects with $R>55$ were excluded. Group 2 now comprised 43 participants. Mean age was 32.8 years, ranging from 23 to 42 years, and mean years 
of education was 12.8 years with a range from 12 to 16 years. There were 16 men $(37 \%)$ and 27 women $(63 \%)$.

Group 3 comprised 25 of 29 consecutive patients from a County Council outpatient unit set up to offer dynamic psychotherapy to severely disturbed patients with personality disorders. The referred patients had proved to be difficult to treat within the framework of the County Council psychiatric service, and in most cases different treatments had been tried without satisfactory results. Many of the patients had a history of social maladaptation and of different kinds of acting out. However, the referring units still regarded the patients as possible to treat and in need of psychodynamic therapy. There were six men (24\%) and 19 women (76\%), and ages ranged from 20 to 44 years, with a mean of 30.8. Mean years of education was 12.9, ranging from nine to 18 years. All patients had at least one DSM-III-R diagnosis on Axis II, and mostly at least one diagnosis on Axis I (APA, 1987). Structurally, all patients in the group were assessed as organised on a borderline personality level (Kernberg, 1975). One patient where the therapy had already started when the Rorschach was administrated, and two patients that turned out to be psychotic and in need of heavy medication were excluded. Moreover, to make group 3 similar to group 1 and 2 , one subject with more than 20 years of education was excluded.

There were more men in group 2 than in group 1 and group 3 . However, for the three samples combined there were no significant differences in CS scores between men and women as calculated with the Median test.

Study 3 (Paper II), CS and two personality disorder groups with different therapy suitability, compared two groups of patients with personality disorder according to DSM-IV (APA, 1994). The same group from the psychotherapy unit for personality-disordered patients as in Study 2 was compared with a new sub-sample from the 69 patient group of the S-COMPAS. The selection of the new sub-sample was carried out in the following way. All patients with at least one diagnosis on Axis II according to DSM-IV (APA, 1994) were selected first. Then three subjects older than 46 years and two subjects with $\mathrm{R}>55$ were excluded. The sample then comprised 21 female $(60 \%)$ and 14 male (40\%) patients, mean age was 33.3 years, ranging from 23 to 46 years, and mean years of education was 12.0 years with a range from 8 to 16 years. Because there were more males in the second group, the medians for the CS scores were compared using the Median test. However, there were no significant differences between males and females. The overlap between the S-COMPAS sub-samples in Study 2 and Study 3 comprised 26 participants.

Study 4 (Paper III), Pre-treatment CS, post-treatment Dynamic Capacity 
and Patient Satisfaction, comprised participants from the 69 S-COMPAS sample. Ratings of Patient Satisfaction after termination of therapy were obtained from 48 therapy patients. One patient had been in psychodynamic long-term group psychotherapy ( $\geq 2$ years), while 47 patients had received long-term ( $\geq 2$ years) individual dynamic psychotherapy (of these patients six had received body-oriented therapy and three symbol drama, one art therapy, and the other patients standard individual psychotherapy). There were 17 men and 21 women, and mean age was 35.5 years, ranging from 23 to 62 years. Five of the participants had finished comprehensive school, 30 had finished senior high school, and 13 had a university education. The drop-out group consisted of 21 patients. Age, level of education, number of diagnoses on Axes I and II according to DSM-IV (APA, 1994), and pre- and post-therapy ratings of Dynamic Capacity did not differ significantly between the drop-out group and the 48 participants. However, two of the CS variables, R and F\%, were significantly higher in the drop-out group. The most plausible explanation for the drop out is the voluntary nature of the situation after termination of therapy.

Post-therapy therapist ratings of Dynamic Capacity were obtained for 44 patients in the Nygren S-COMPAS 69 cases sample. There were 20 men and 24 women. Two patients had received group therapy and 42 had received longterm individual dynamic psychotherapy (of these therapies five were body-oriented and three were symbol drama). Mean age was 35.7 years, ranging from 23 to 62 years. One participant had finished comprehensive school, 32 had finished senior high school, and 11 had a university education. The drop-out group comprised 25 patients. However, there were no significant differences between the drop-out group and the participants concerning age, level of education, number of diagnoses on Axes I or II according to DSM-IV (APA, 1994), and the selected CS variables. The most plausible explanation for the drop out is again the voluntary character of the post-treatment therapist ratings.

The overlap between drop-out for post-therapy Patient Satisfaction ratings and post-therapy therapist rated Dynamic Capacity consisted of 14 patients. However, age, level of education, number of diagnoses on Axes I and II according to DSM-IV (APA, 1994), and pre-therapy ratings of Dynamic Capacity did not differ significantly between the drop-out overlap group and the 55 participants for whom ratings of Patient Satisfaction and/or Dynamic Capacity were obtained. 


\section{Assessments}

The assessments in the present studies comprised clinical ratings and the Rorschach according to the CS. In Study 1 and Study 4 the focus was on correlations between clinical ratings and CS variables. The ratings concerned variables related to therapy suitability, and, as mentioned earlier, in both studies experienced clinicians carried out the ratings independently of the Rorschach; they were not informed about and did not have access to any written material about the results from the Rorschach. Table 3 presents an overview of the measures in this research.

Table 3. Assessments in Study 1-4.

\section{Measures}

Study 1 (Paper I) Rorschach according to the CS, and pre-treatment Dynamic Capacity and Ego Strength as represented by experienced clinicians' ratings.

Study 2 (Paper II) Rorschach according to the CS.

Study 3 (Paper II) Rorschach according to the CS.

Study 4 (Paper III) Rorschach according to the CS, and Patient Satisfaction as represented by patients' evaluation of psychotherapy

and

Rorschach according to the CS, and Dynamic Capacity as represented by therapists' post-treatment ratings.

\section{Clinical ratings}

In Study 1, Pre-treatment CS, pre-treatment Dynamic Capacity and Ego Strength, the clinical ratings of Dynamic Capacity and Ego Strength were carried out by 15 experienced clinical interviewers, who were students on an advanced psychotherapy training program. Seven 7-point items aiming at Ego Strength, and three 7-point items relating to aspects of Dynamic Capacity were formulated by the author in co-operation with the program's teachers and supervisors. The rating form was supplemented by extensive descriptions of the meaning of the rating variables. Moreover, the patients were thoroughly discussed in supervisory teams before the carrying out of the ratings. The ratings 
were carried out independently of the Rorschach; neither the supervisors nor the students had any knowledge of the Rorschach results.

In Study 4, Pre-treatment CS, post-treatment Dynamic Capacity and Patient Satisfaction, three 6-point rating variables regarding the suitability for psychotherapy were included. The ratings were carried out by 38 therapists, who rated their patients before the onset of therapy and after its completion. The post-therapy ratings concerning the patient's Dynamic Capacity were carried out when it is reasonable to assume that the therapists had good knowledge of their patients. All therapists were fully qualified private practitioners who did not work at the referring clinics and who did not take part in the assessment of the patients in the referring psychiatric units. Neither the results of the Rorschach assessment nor any material from the patients' files was available to the therapists.

\section{Rorschach administration and scoring}

The Rorschach was administered and scored according to the CS in all the studies. The author, an experienced practitioner and instructor of the CS, carried out the administration and the scoring of the Rorschach for all therapy applicants in the training unit (Study 1). The author also carried out the majority (23 of 25) of the administration and scoring of the Rorschach for the participants from the psychotherapy unit for severe personality disorders (one of the samples in Study 2 and 3). However, for the 69 participants selected from the S-COMPAS, the author and 12 other psychologists with extensive experience of, and advanced education in the CS, administered and scored the Rorschach. One psychologist tested 18 participants, one tested 15, one 12, two psychologists tested five participants each, three tested three participants, one tested two participants, and finally three psychologists tested one participant each.

To assess the reliability of the CS scoring, intraclass correlations (ICC) for protocol-level agreement were calculated using the statistical software Rorschach Research Utilities version 0.9.2, developed by Harald Janson (Janson \& Olsson, 2001).

For the Rorschach protocols from the psychotherapy applicants at the psychotherapy training unit ( $n=52$, Study 1 ), reliability was calculated for 20 protocols. For the protocols from the therapy unit for severe personality disorders (a sample in Study 2 and 3), reliability was calculated for 10 protocols. Finally, from the 69 protocols selected from the S-COMPAS Rorschach database, ICC was calculated for 21 protocols. 
Table 4. ICC values for responses summed for whole Rorschach protocols, for the psychotherapy training unit sample, the sample from the S-COMPAS, and for the sample from the therapy unit for severe personality disorders.

\begin{tabular}{lccc}
\hline CS score & $\begin{array}{c}\text { Psychotherapy } \\
\text { training unit } \\
(n=20)\end{array}$ & $\begin{array}{c}\text { S-COMPAS } \\
(n=21)\end{array}$ & $\begin{array}{c}\text { Therapy unit for } \\
\text { personality disorders } \\
(n=10)\end{array}$ \\
EA & .89 & .91 & .97 \\
FC & .79 & .78 & .79 \\
YF & .57 & .56 & .79 \\
Y & .79 & - & - \\
m & .68 & .86 & .91 \\
SumT & .83 & .94 & .74 \\
D Score & - & .75 & .76 \\
Blends & .86 & .91 & .97 \\
CDI & .65 & .73 & .71 \\
Zf & .96 & .98 & .98 \\
F \% & .82 & .92 & .95 \\
MQo & .72 & .65 & .89 \\
WSUM6 & .84 & .84 & .86 \\
X-\% & .80 & .62 & .52 \\
S & .92 & .94 & .98 \\
FrrF & 1.00 & .93 & .92 \\
FD & .72 & .74 & .86 \\
AG & .74 & .84 & .74 \\
COP & .71 & .77 & .89 \\
PER & .78 & .55 & .88 \\
& & & \\
\hline
\end{tabular}

Table 4 presents the ICC values for whole protocols for the three samples of the present research. According to the recommendations by Cicchetti (1994), the reliability of the scoring was interpreted as acceptable for all CS variables selected in this research. ICC values less than .40 are regarded as poor, between .40 and .59 as fair, between .60 and .74 as good, and over .74 as excellent (Cicchetti, 1994).

The lowest ICC values .52-.57, obtained for YF, X-\%, and PER in one or two of the samples are fair values, and the ICC values for the remaining CS variables are within the range good to excellent. 
The CS and assessment for dynamic psychotherapy

Table 5. Summary of the statistical procedures in Study 1-4.

\begin{abstract}
Study 1 (Paper I) Correlations between CS variables and clinical ratings of Dynamic
Capacity and Ego Strength were calculated. CS variables between patients selected and patients not selected for psychotherapy were compared using independent samples $t$ test.

Study 2 (Paper II) Three groups were compared using Kruskal-Wallis 'ANOVA'. Pair-wise analyses of groups were made using the Exact Mann-Whitney $U$ test.

Study 3 (Paper II) Two groups of psychotherapy patients were compared using the Exact Mann-Whitney $U$ test and Fisher's Exact $\chi^{2}$ test for CS variables where cut off values were specified.

Study 4 (Paper III) Correlations between CS variables and post-therapy Patient Satisfaction were calculated. Not satisfied and medium to highly satisfied patients were compared using the independent samples $t$ test. Correlations between CS variables and post-therapy Dynamic Capacity were calculated.
\end{abstract}

\title{
Statistical procedures
}

The correlations calculated in the present studies were the Pearson and the Spearman correlation, and partial correlation. Groups were compared using the independent samples $t$ test and the Exact Mann-Whitney $U$ test, and in Study 3 comparisons between groups for CS scores with cut-off values specified were carried out using Fisher's Exact $\chi^{2}$ test. In Study 2, three groups were compared using the Kruskal-Wallis non-parametric 'analysis of variance' (Hays, 1973). Because the expected direction of the correlations and group differences was formulated a priori, the significance tests were one-tail except for the Kruskal-Wallis 'analysis of variance' where the significance test was two-tail followed by pair-wise comparisons based on the results.

Study 1 (Paper I), Pre-treatment CS, pre-treatment Dynamic Capacity and Ego Strength. Correlations between the selected CS variables and the criterion variables were calculated. However, for many of the CS scores the influence of number of responses $(\mathrm{R})$ had to be controlled for. According to the recommendations by Viglione (1995) and Curran, West, and Finch (1996), the parametric partial correlation was used if the skew of the CS variables was $\leq 2$ or the kurtosis $\leq 7$. When partial correlation could not be used, the nonparametric Spearman correlation was calculated controlling for number of responses by dividing the $\mathrm{CS}$ variable with $\mathrm{R}$. The significance tests for all the predicted correlations were one-tailed. On the assumption that the CS variables should differ between the applicants selected for psychotherapy and those not selected in 
the same direction as was predicted for the correlations, the comparison between these two groups was carried out using one-tailed independent samples t-test. Effect sizes were calculated with Cohen's $d$ using pooled variance and adjusted for unequal sample sizes.

Study 2 (Paper II), CS and three groups differing in therapy suitability. The comparison of the CS variables in three groups was carried out using the Kruskal-Wallis 'analysis of variance' (Hays, 1973) (two-tail), a nonparametric method equivalent to one-way ANOVA. Pair-wise analyses based on the results of the 'analysis of variance' were then carried out with the nonparametric Exact Mann-Whitney $U$ test (one-tail). The selection of CS scores for these pair-wise comparisons was carried out in the following way. For the CS scores that differed with $p<0.1$ in the predicted direction, pair-wise comparisons were made between group 3 and group 1 , and between group 3 and group 2 . This kind of pair-wise comparisons were also carried out when the mean rank in the Kruskal Wallis 'ANOVA' indicated that group 3 differed markedly from group 1 or 2 , even if $p \geq 0.1$.

Study 3 (Paper II), CS and two personality disorder groups with different therapy suitability, compared two groups of patients with personality disorder according to DSM-IIIR or DSM-IV(APA, 1987; 1994), using the Exact MannWhitney $U$ test. Comparisons concerning CS variables with specified cut-off values were made using Fisher's Exact $\chi^{2}$ test. Because the groups in Study 2 and 3 were made similar with respect to $\mathrm{R}$, no separate control for $\mathrm{R}$ was needed.

Study 4 (Paper III), pre-treatment CS, post-treatment Patient Satisfaction and Dynamic Capacity. Correlations between the selected CS variables and the criterion variables were calculated in the same way as in Study 1, and the influence of $\mathrm{R}$ was also controlled in the same way as described in Study 1. Moreover, the Patient Satisfaction variable was partitioned into two groups, one not satisfied and one medium to highly satisfied, and CS variables in the two groups were compared with the independent samples $t$ test (one-tail).

As mentioned, the statistical tests in the present studies were one-tail and the significance level was .05. Despite the great number of significance tests, correction for mass significance was not used. The reason was that a separate prediction was formulated for each of the selected CS variables and then tested in different studies with different designs and to a great extent comprising different participants. For the interpretation of the results the degree of consistency was considered important. 
The CS and assessment for dynamic psychotherapy

Table 6. Number of hits in the predicted direction in relation to number of possible hits (in each study and totally) presented for the four studies.

\begin{tabular}{|c|c|c|c|c|c|c|}
\hline CS variable & Study 1 & Study 2 & Study 3 & $\begin{array}{c}\text { Study } 4 \\
\text { Patient } \\
\text { Satisfaction }\end{array}$ & $\begin{array}{c}\text { Study } 4 \\
\text { Dynamic } \\
\text { Capacity }\end{array}$ & $\begin{array}{l}\text { Sum of hits by } \\
\text { number of } \\
\text { possible hits }\end{array}$ \\
\hline \multicolumn{7}{|c|}{ Control and stress tolerance: } \\
\hline EA & $3 / 3^{a}$ & $3 / 3^{d}$ & $2 / 2^{\mathrm{e}}$ & $0 / 2^{f}$ & $1 / 1^{g}$ & $9 / 11$ \\
\hline FC & $3 / 3^{a}$ & $0 / 1^{c}$ & $0 / 1^{e}$ & $0 / 2^{f}$ & $1 / 1^{g}$ & $4 / 8$ \\
\hline YFY & $3 / 3^{a}$ & $0 / 1^{c}$ & $0 / 1^{e}$ & $0 / 2^{f}$ & $0 / 1^{g}$ & $3 / 8$ \\
\hline $\mathrm{m}$ & $0 / 2^{b}$ & $0 / 1^{c}$ & $0 / 1^{e}$ & $0 / 2^{f}$ & $0 / 1^{g}$ & $0 / 7$ \\
\hline SumT & $0 / 2^{b}$ & & $0 / 1$ & $0 / 2^{f}$ & $0 / 1^{g}$ & $0 / 6$ \\
\hline Blends & $2 / 3^{a}$ & $2 / 3^{d}$ & $1 / 1^{\mathrm{e}}$ & $2 / 2^{f}$ & $1 / 1^{g}$ & $8 / 10$ \\
\hline DScore & & & $1 / 1$ & $2 / 2^{f}$ & $1 / 1^{\mathrm{g}}$ & $4 / 4$ \\
\hline CDI positi & tive & & $1 / 1^{\mathrm{e}}$ & & & $1 / 1$ \\
\hline \multicolumn{7}{|c|}{ The cognitive triad: } \\
\hline $\mathrm{Zf}$ & $2 / 2^{b}$ & $1 / 3^{d}$ & $1 / 1^{e}$ & $1 / 2^{f}$ & $0 / 1^{g}$ & $5 / 9$ \\
\hline $\mathrm{F} \%$ & $2 / 3^{a}$ & $1 / 3^{d}$ & $1 / 2^{e}$ & $2 / 2^{f}$ & $1 / 1^{\mathrm{g}}$ & $7 / 11$ \\
\hline MQo & $2 / 2^{b}$ & $2 / 3^{d}$ & $1 / 1^{\mathrm{e}}$ & $0 / 2^{f}$ & $1 / 1^{g}$ & $6 / 9$ \\
\hline WSUM6 & $0 / 2^{b}$ & $0 / 1^{c}$ & $0 / 2^{e}$ & $0 / 2^{f}$ & $0 / 1^{g}$ & $0 / 8$ \\
\hline$X-\%$ & $0 / 2^{b}$ & $0 / 1^{c}$ & $0 / 2^{\mathrm{e}}$ & $0 / 2^{f}$ & $0 / 1^{g}$ & $0 / 8$ \\
\hline \multicolumn{7}{|c|}{ Affective features: } \\
\hline$S$ & $0 / 2^{b}$ & $0 / 1^{c}$ & $0 / 2^{\mathrm{e}}$ & $0 / 2^{f}$ & $0 / 1^{g}$ & $0 / 8$ \\
\hline \multicolumn{7}{|c|}{ Self and interpersonal perception: } \\
\hline FrrF & $0 / 2^{b}$ & $0 / 1^{c}$ & $0 / 1^{\mathrm{e}}$ & $0 / 2^{f}$ & $0 / 1^{g}$ & 0/7 \\
\hline FD & $0 / 2^{b}$ & $1 / 3^{d}$ & $1 / 2^{e}$ & $0 / 2^{f}$ & $0 / 1^{g}$ & $2 / 10$ \\
\hline AG & $0 / 2^{b}$ & $2 / 3^{d}$ & $1 / 1^{\mathrm{e}}$ & $1 / 2^{f}$ & $0 / 1^{g}$ & $4 / 9$ \\
\hline COP & $0 / 2^{b}$ & $2 / 3^{d}$ & $0 / 1^{e}$ & $0 / 2^{f}$ & $0 / 1 \mathrm{~g}$ & $2 / 9$ \\
\hline PER & $1 / 2^{b}$ & $0 / 1^{c}$ & $0 / 1^{e}$ & $0 / 2^{f}$ & $0 / 1^{g}$ & $1 / 7$ \\
\hline
\end{tabular}

Note. Tendencies ( $p$ between .050 and .100) reported in the studies are not included.

a Correlations between CS variable, Dynamic Capacity and Ego Strength, and independent samples t-test of applicants selected and not selected.

b Correlations between CS variable and Dynamic Capacity or Ego Strength, and independent samples $t$ test of differences between applicants selected and not selected for therapy.

c Three groups compared.

d Three groups compared, and also pair-wise comparisons based on results from threegroup comparison.

e Comparison between two groups of patients with personality disorder. For EA, F\%, WSUM6, X-\%, S, and FD also cut-off values. For SumT, the D Score, and CDI only cutoff values.

f Correlation between CS variables and Patient Satisfaction and $t$ test of differences between patients not satisfied and patients medium to highly satisfied.

g Correlation between CS variables and Dynamic Capacity. 


\section{RESULTS}

To give an overview, Table 6 presents a summary of the results of the studies. Results with $p>.05<.10$ are regarded as tendencies and therefore mentioned in the text. However, in Table 6 no tendencies are included in the presentation.

Study 1 (Paper I), Pre-treatment CS, pre-treatment Dynamic Capacity and Ego Strength. In accordance with the predictions EA, FC, Blends, Zf, and MQo correlated positively and YFY negatively with Dynamic Capacity ratings, and for AG there was an almost significant $(p=.051)$ correlation in the predicted direction. EA, FC, and Blends correlated positively and YFY and F\% negatively with Ego Strength ratings. EA, FC, YFY, Zf, MQo, and F\% also differed significantly between the applicants selected and those not selected for psychotherapy. The effect sizes for the significant results ranged from 0.49 to 0.68 .

Study 2 (Paper II), CS and three groups differing in therapy suitability. The Kruskal-Wallis test was significant for EA, Blends, MQo, and COP. In the pairwise comparisons between group 1 and 3 , and between group 2 and 3 the results for EA and AG were significant in the predicted direction in both comparisons. For Blends, F\%, Zf, and COP the result was significant in one group comparison with a trend in the predicted direction for the other, and for MQo and FD the results were significant in one of the pair-wise group comparisons.

Study 3 (Paper II), CS and two personality disorder groups with different therapy suitability. The difference between the two groups of patients with personality disorder was significant in the predicted direction according to the Mann Whitney $U$ test for EA, Blends, Zf, F\%, MQo, FD, and AG. For the comparison between the two groups concerning CS variables with cut-off values using Fisher's exact $\chi^{2}$, the results for EA, the D Score, and the CDI were significant.

Study 4 (Paper III), Pre-treatment CS, post-treatment Patient Satisfaction and Dynamic Capacity. Patient Satisfaction correlated significantly in the predicted direction with the D Score, Blends, F\%, and Zf. For AG there was a tendency in the predicted direction.

For Dynamic Capacity the correlations were significant in the predicted direction for EA, FC, the D Score, Blends, F\%, and MQo. For Zf and COP there were tendencies in the predicted direction.

The CS variables in a group of patients with low satisfaction $(n=17)$ were 
compared with those in a group with medium to high satisfaction $(n=31)$ using the independent samples $t$ test and the Exact Mann-Whitney $U$ test. The results were significant in the predicted direction for Blends, the D Score, F\%, and AG, and for Sum T, Zf, and MQo there were tendencies in the predicted direction. The effect sizes ranged from 0.47 to 0.73 .

Because the D Score was not included in the research until Study 3, there is a risk that the number of reported hits in relation to number of possible hits gives an overly positive impression. Therefore, post hoc calculations of the correlations between the $\mathrm{D}$ Score and Dynamic Capacity were calculated for the sample in Study 1 . The correlation was not significant $(0.21, p=.125)$, and opposite to what would have been predicted.

\section{DISCUSSION}

The present thesis comprises four studies focusing upon selected CS variables and their relation to suitability for psychodynamic psychotherapy. The selection of CS variables was based on the assumption that, according to the interpretations given to them in the CS, the variables should be related to aspects of ego functioning in turn related to suitability for psychodynamic psychotherapy.

The predictions in the four studies were formulated on the same conceptual ground and the results in the different studies were expected to be in the same direction. The results from an earlier study did not influence the predictions in subsequent studies. This sometimes led to predictions that contradict what would have been formulated if the predictions had been based on the actual results observed in the earlier studies.

With a few exceptions, the CS variables are the same in the studies; 16 of the 19 selected variables were included in all four studies. The D Score was not included in Study 1 and 2, Sum T was not included in Study 2, and the CDI was only included in Study 3. The reasons for these decisions were presented earlier in connection with a description of the CS variables selected. For SumT and CDI there were good reasons for the decisions, but for the D Score there is not the same degree of rationality.

The Rorschach was methodologically independent of the ratings in Study 1 and Study 4. The groups in Study 2 and Study 3 were construed to differ in one aspect, therapy suitability. The Rorschach did not influence the selection 
of subjects to the groups. It was possible to make predictions for the CS variables concerned and to test predictions without any flaws.

The design of the group comparisons differs from the most common strategy of group comparisons in Rorschach research. The usual strategy has been to contrast two or more patient groups, often quite multifaceted without having clear hypotheses grounded in theory and/or earlier empirical results. The Rorschach differences identified this way have then been attributed to the known difference between the groups, that is, predictions have not been formulated and motivated before the comparisons of CS variables were carried out (Exner, 1993; Dies, 1995). This approach renders it difficult to know whether the indicated difference between the groups is crucial, in turn making it difficult to draw conclusions concerning the differences in Rorschach variables. However, although the design in Study 2 and 3 permitted testing of clearly formulated hypotheses, there may be other explanations of the obtained significant differences than those implied in the predictions; what is attained is that the certainty of the conclusions becomes stronger.

One central question permeates the four studies in this thesis: 'Are interpretations commonly associated with the CS supported by their relation to clinical data representing suitability for dynamic psychotherapy to which they should on conceptual grounds be related?'

\section{Pattern of the results and agreement with earlier research}

When interpreting the results for the CS variables that received positive results in this thesis, the risk of mass significance must be considered, i.e. the risk that the results happened to show statistical significance when, in effect 'there is nothing'. There is also the problem that the studies are not perfectly independent. As can be seen from Table 2, there is a degree of overlap concerning participants. However, predictions were formulated for each variable separately, the predictions for one variable were tested several times, and repeated tests of the predictions were considered to be of central importance for the interpretation of the results.

For eight of the 18 (CDI not counted because it was only included in Study 3) selected CS variables, the verifications of the predictions are deemed to be too consistent to be chance results. Moreover, the results cannot possibly be explained by the overlap of participants in the different studies. 
Four of the supported variables, EA, FC, Blends, and the D score concern control and stress tolerance; EA and Blends received strong consistent support, whereas the support for the D score and FC was somewhat less consistent. The control capacity related to availability of psychological resources, interpreted to be indicated by EA, mostly indicates an asset. However, when the capacity indicated by EA overshadows the experience of discomfort and anxiety, the control capacity may instead become a hindrance to psychological development and growth. According to the interpretations in the CS, positive values of the D Score indicate this kind of less adaptive, probably ego syntonic control aiming at avoiding discomfort and anxiety. The results in the present studies are in accordance with these interpretations of EA and the D Score.

The verifications of the prediction that Blends should be positively related to Dynamic Capacity are in accordance with the results by Alpher et al. (1990) and by laBarbera and Cornsweet (1985), and the results for the D Score (EAes) as negatively related to Dynamic Capacity and Patient Satisfaction are in accordance with results by LaBarbera and Cornsweet.

Three of the clearly supported CS variables, F\%, Zf, and MQo, concern information processing, ideation, \& mediation. Zf and MQo are interpreted to relate to a dynamic type of processing characterised by ambitiousness, imaginative capacity, and flexibility, whereas F\% is interpreted as indicating a more rigid processing style. The results for $\mathrm{Zf}$, and $\mathrm{F} \%$ are in accordance with the results by Alpher et al. (1990) and by LaBarbera and Cornsweet (1985). Human movement responses which received strong support in this thesis, were not supported in the study by Alpher et al. However, predictions were only formulated for responses of ordinary form, MQo, in the present research, while Alpher et al. (1990) included all M responses, that is, they also included M responses of unusual and poor form quality, and this may explain the negative result in their study. Moreover, the positive results for MQo are in accordance with findings concerning the RPRS (Klopfer et al., 1954) where human movement is a part; RPRS has shown a positive relation to psychotherapy outcome (Garfield, 1994; Meyer, 2000; Meyer \& Handler, 1997, 2000). The conclusion that human movement responses of good form are an important score seems well grounded.

Finally, one of the selected CS variables concerning self \& interpersonal perception, AG, received support. However, for the remaining variables from this cluster the results were not positive. In this thesis, AG is interpreted as indicating forcefulness rather than a more hostile kind of aggressiveness, and the positive results for AG are in agreement with the finding by Hilsenroth et al. 
(1995), that patients terminating psychodynamic psychotherapy prematurely had fewer AG and more COP than had those remaining in treatment.

Considering the importance of replication of the results for the single variables in the different studies, the results for YFY, FD, COP, and PER are too weak to be regarded as convincing. Moreover, for six of the $18 \mathrm{CS}$ variables no predictions were verified; the results for m, SumT, WSUM6, X-\%, S, FrrF were decisively negative. Thus, for 10 of 18 selected CS variables the combined results from the four studies in this thesis are considered negative. Three of these 10 variables, YFY, $\mathrm{m}$, and SumT, are interpretated to be related to different aspects of anxiety and four, FD, FrrF, COP, and PER concern the way in which the patient experiences him/herself and others. Finally, the results were negative for WSum6 and X-\%, interpreted to relate to thought disturbances and deficient reality testing, and for $S$, where higher values are interpreted to indicate a negativistic oppositional set. Thus, although the relations between SumT and $m$ and the Capacity for Dynamic Process Scale were positive in the Alpher et al. study, these variables did not receive support in the present studies. The negative results in this research cast doubt on the value of $\mathrm{m}$ and SumT in assessment for dynamic psychotherapy. However, more research is needed to reach a reliable conclusion especially concerning $m$ that is also one of the scores in the RPRS (Klopfer et al., 1954).

To sum up, in the studies in this thesis the most consistent results were obtained for the CS variables EA, Blends, Zf, F\% and MQo. Although less consistent, the results for FC, the D score, and AG were also in agreement with the predictions, and were interpreted as positive. The results are interpreted to demonstrate that these CS variables are related to suitability for dynamic psychotherapy. On the other hand, the results for m, SumT, WSUM6, X-\%, S, and FrrF were negative, and for YFY, FD, COP, and PER the hits were too few to permit a positive interpretation.

\section{Interdependence of supported CS variables}

EA, MQo, FC, and the D Score that received support in this research are not independent of each other in the CS structural summary; the D Score is calculated on the raw score EA-es, and EA in turn includes FC and MQo. However, FC and MQo are only two of many variables constituting EA and the D Score, and they also have their interpretations as single variables in the CS. 
It follows, however, that the variables that have received support in the present studies cannot be expected to be uncorrelated. The inter-relations among the variables that received substantial support were analysed post-hoc using principal component analyses with Varimax rotation, including R, EA, FC, the D Score, Blends, F\%, Zf, MQo, and AG. The subjects comprised the participants of the four studies $(n=142)$. The skew of the CS variables varied between -0.360 and 1.527 and the kurtosis between 0.104 and 4.457. Three components with an Eigenvalue $>1$ were obtained. Six of the CS variables have high loadings on one component and low on the other components. EA, FC, and Zf load mainly on the first component, F\% and AG on the second, and the D Score on the third. For Blends and MQo the picture is less clear; Blends load on the first component but also on the second, and MQo loads mainly on the third component but also to some extent on component one. Thus, Blends and MQo were less factor specific and did not fit a simple structure, but on the whole the results are promising as low correlations between predictors increase their contribution as a group to predictions.

Although the component analysis is only tentative, the components are interpreted in accordance with the CS conceptualisations of them. The first component integrates control and stress tolerance aspects with cognitive and emotional aspects. It indicates ambitiousness, motivation and capacity for deliberate coping strategies ( $\mathrm{Zf}$ and $\mathrm{EA}$ ), cognitively directed and controlled affective experiences (FC), but also capacity for inner directed imagination and fantasy and dealing with complex, often emotional, experiences (MQo, and Blends). The second component seems to indicate the capacity to be assertive and forceful (AG) while not defensively narrowing and simplifying the experience field (indicated by F\% and Blends). The third component, dominated by the $\mathrm{D}$ Score, seems to be related to control of anxiety that is a hindrance in dynamic psychotherapy. The positive loading of MQo on this component may imply that MQo can be involved in psychological defensive processes that are not an advantage in psychodynamic psychotherapy. This problem needs to be considered in research and practice.

\section{Comments on some single CS variables}

As made clear earlier, the D Score was not included until Study 3, and to make the picture of the D Score complete post hoc correlations for Study 1 were cal- 
culated; the results were negative. One possible explanation is that at the psychotherapy unit, the site of Study I, there was perhaps too great a tendency to avoid patients suspected of failing control during therapy. This explanation fits with the fact that predictions for YFY, interpreted as indicating anxiety and experience of helplessness in relation to the surrounding world, and hypothesized to be negatively related to therapy suitability, were only confirmed in Study 1 . The risk for this kind of anxiety and the risk for lessened control during therapy may have been regarded as more troublesome at the training unit than at the County Council units. At the training unit, it was important that therapy patients could stick to a two-year twice a week therapy without acting out by often failing to come or by prematurely terminating therapy.

Some of the CS variables that did not receive support in the present studies merit further research before conclusions about their value in assessment for dynamic psychotherapy can be certain. For diffuse shading responses and for colour responses the predictions did not encompass all varieties of form dominance. The influence of form dominance on these variables in assessment for dynamic psychotherapy should be better elucidated, before drawing any conclusions about their value as psychotherapy indicators. For example, a high number of FC (selected in this research) can indicate too strong restraint of emotional expression. In the CS, FC is interpreted in relation to the non-form dominant colour responses, $\mathrm{CF}$ and $\mathrm{C}$. Research into different combinations of $\mathrm{FC}$ and $\mathrm{CF}$ and $\mathrm{C}$ in assessment for therapy could be informative.

On the whole, research into the implications for psychotherapy assessment of different levels of form dominance of the CS variables that are parts of EA and es should be informative.

\section{Structural and qualitative analysis of the Rorschach}

As in the studies by Alpher et al. (1990) and LaBarbera and Cornsweet (1985), the present research focused on a selection of CS summary scores. Content aspects, combinations of CS variables or analysis of qualitative aspects of the patients' CS responses as a platform for more global assessment of suitability for dynamic psychotherapy, were not included. Since the advent of the Comprehensive System (Exner, 1991, 1993, 2003), research has focused mostly on discrete codes, while research using a global-holistic approach has become rare. Janson and Stattin (2003) pointed out that Rorschach research has shifted be- 
tween the objective coding of discrete response elements and global-holistic ratings. Although both approaches are valuable, Janson and Stattin also called attention to the important fact that clinicians regularly combine discrete codes into meaningful categories, and that the dichotomy between single coded data and a global-holistic approach, often forthcoming in research, may seem artificial to clinicians. Costello (1999) and Karon (2000) emphasised the importance of interpretations of qualitative clinical data in the Rorschach, and Karon warned that formal scoring systems lack valuable information. Acklin (1992) and Weiner (2000a) emphasised the importance of an integrative approach in the interpretation of Rorschach. However, in accordance with what Weiner (1996, 2001) contended, for an approach using both the structural and the qualitative aspects of the CS responses in psychotherapy assessment, the clinician should be guided by knowledge about which CS variables are valid for that specific purpose. For CS variables that are supported as indicators for dynamic psychotherapy, there is need to seek a deeper understanding about the value of systematic combinations of the variables into meaningful entities and greater knowledge about the integration of structural coding and qualitative aspects.

\section{Implications for clinical psychotherapy assessment}

The present studies took place in naturalistic and clinically relevant circumstances. Therefore, the results are ecologically relevant and might be of value in clinical psychotherapy assessment. When making such assessment it is advisable not to look merely for the kind of pathological problem the patient has and then to select the method assumed to be clinically effective in treating that problem. Such an approach, often represented by the use of the DSM system as a main instrument in therapy planning, is an oversimplification of the clinical reality; diagnostic labels create a deceptive illusion of homogeneity (Roth \& Fonagy, 1996). Moreover, the research concerning suitability for psychodynamic psychotherapy, mentioned in the introduction, supports the value of variables other than formal diagnosis. Motivation, psychological mindedness and object relations stand out as central and desirable assets in dynamic psychotherapy.

However, as pointed out by Valbak (2003), the variables mostly regarded as central in assessment for dynamic psychotherapy are theoretically grounded and defined in abstract terms. This can be problematic in applied psycho- 
therapy assessment. Although descriptions of the variables such as those used in Study 1 are helpful, the abstract character remains a difficulty. What is assessed will be dependent on the therapist's interpretations of what the patient says, in terms of the variables assessed. The Rorschach variables on the other hand are based directly on the patient's description of what he/she sees when presented with the test stimuli, and the interpretation is not made until the responses are coded. The interpretations of CS variables in terms of psychodynamic theory can therefore be regarded as grounded in data obtained without individual therapist interpretations. For CS variables that have been validated as useful in psychotherapy assessment, their complementary value is grounded in this difference in starting-point.

The CS structural variables that have obtained support in research may inform about aspects that are difficult to catch during the assessment interview. Their value is not to asses the patient's pathology; instead, they should complement the interview in considering the patient's pattern of assets and liabilities with regard to psychodynamic psychotherapy. Such information can also be valuable in situations when a therapy does not work out as expected. The Rorschach administered before therapy can be reconsidered during therapy, and sometimes a Rorschach test used during therapy can help therapist and patient to find more constructive ways to handle problems.

A few examples may be illuminating. It is sometimes difficult to discern limited availability of psychological resources and/or low flexibility during assessment interviews, especially if the patient is a well-behaved and verbally well endowed person. These are liabilities that can be indicated by low values of EA and high F\% values. Such complementary findings from the Rorschach may help to avoid overly optimistic psychotherapy plans. A tendency to avoid psychic pain may not be revealed until several therapy sessions have passed. Regarding patients using such defensive strategies, a high value of the D Score, known before the start of the therapy, may give invaluable hints for both patient and therapist. Careful planning and adapting of the therapy to the individual patient's capacities can include a strategy to develop or strengthen lacking or weakened capacities that are important for the therapeutic progress early in the therapy. These liabilities may thus themselves become central targets early in therapy with the goal that the patient will benefit more fully from later parts of the therapy.

The clinical implications of some of the negative results in the present research should also be noted. The negative result for reflection responses, FrrF, is important, because being erroneously assessed as narcissistic can have serious 
negative consequences for how a patient will be treated. The results in this thesis imply that reflection responses should not be used as bases for therapeutic decisions. The same seems to hold true for the cognitive difficulties interpreted to be indicated by WSUM6 and X-\% and the negativism interpreted to be indicated by high values of $S$.

The Rorschach is a time-consuming and expensive assessment method. However, if the Rorschach is used in a more focused way than is often the case it should be less time-consuming. As Meyer (2001) pointed out, the clinician who chooses to include the Rorschach in a clinical evaluation of a patient should be able to articulate why this choice is motivated. The Rorschach is a multidimensional method, and as Weiner $(1996,2001)$ contended, individual scores should have their own validity for different purposes. As mentioned earlier, Garb et al. (2002) recommended that only CS variables that have been researched and found valid for a specific question should be used in the applied fields of psychology. Moreover, the questions motivating the use of the Rorschach as a complement to the clinical interview in decisions concerning dynamic psychotherapy, should be clearly formulated in each case. If the focus could be on selected CS scores that have proved to be useful as indicators for psychotherapy, and not on writing lengthy descriptions of the test person's personality, the method would be more effectively adapted to the requested assessment. This should also lessen the risk that statements actually not relevant for psychotherapy planning influence decisions with unfortunate consequences for the patient. However, this also requires correct administration and scoring of the Rorschach, not only in research but also in clinical practice; McGrath (2003) stressed that it is essential to avoid idiosyncrasies in scoring and administration. A psychodynamic psychotherapy is a major and important personal undertaking mainly for the patient but also for the therapist, and the time spent in careful assessment and planning to avoid unintended consequences is worth the costs of time and other resources.

\section{Concluding remarks}

The main weakness of this research is that it was not possible to test the validity and the interrater reliability of the ratings. The rating variables are justified as representatives of dominating clinical reasoning concerning aspects of ego functioning regarded as important for the process and outcome in psychody- 
namic psychotherapy. The rating variables were formulated so as to be natural for the therapists carrying out the ratings and in the situations in which they were used. The criteria in Study 1 were based on very careful clinical ratings and similar to clinical evaluations generally performed in connection with psychodynamic psychotherapy, and in this case carried out under very favourable conditions. The therapists carrying out the post-therapy ratings in Study 4 had good knowledge of the patients based on the preceding fairly long therapies. In addition, the participants in the studies were psychiatric patients in naturalistic conditions. This constitutes a strength that renders the results ecologically valid and possible to generalize to real world dynamic psychotherapy situations. Thus, as McGrath (2001) recommended, the research was adjusted to the clinical realities and all subjects came from clinical populations. The lack of interrater reliability data makes it impossible, however, to estimate the attenuation in the correlations between the CS scores and the clinical ratings.

Although research concerning univariate validity can be considered as a prerequisite for incremental validity and clinical utility (Lilienfeld et al., 2000; Meyer, 2000), there is a need for deeper understanding of the incremental value of the CS variables supported in this thesis. Univariate validity refers to the association between a single predictor and a criterion, whereas incremental validity refers to the extent to which an instrument contributes valid and unique information over and above other instruments. The present studies gave consistent support to the univariate validity of some CS scores but it did not inform about the incremental validity of the CS variables for psychotherapy assessment.

However, it seems that for the patients in Study 2 and 3 referred to the psychotherapy unit set up to treat severely disturbed patients with personality disorders, the Rorschach might have provided valuable information, if it had been administered earlier. Most of these patients had a long history of unsuccessful treatments although they had been interviewed and assessed many times in connection with psychiatric treatment decisions. Moreover, as Valbak (2003) pointed out, careful and comprehensive assessments are most important when assessing patients who are not obviously suitable for therapy. These are often patients with a personality disturbance and evident psychic pain, who do not have a well-developed capacity for object relations and who do not stand out as psychologically minded and strongly motivated for the demands of the dynamic psychotherapy process. The value of the Rorschach as a complement to the clinical interview for this group of patients merits special attention, not focusing primarily on the capacity of the CS to differentiate these patients from 
other more well-functioning groups of patients, but on the relation to therapy process and outcome.

The selection of CS variables was mostly focused on single CS variables and not on ratios and combination of codes. The main reason for this was a striving for simplicity and the need to limit the number of the predictions. However, to obtain a more complete knowledge about Rorschach according to the CS and psychodynamic psychotherapy, further research is needed. Research focusing on CS and therapy assessment, process and outcome, and research including composite CS variables, content variables, and qualitative aspects is desirable. Moreover, with regard to psychotherapy planning, there is a need for more research concerning the validity of CS variables indicating problem areas and therapy goals.

Thus, while recognising the limitations of the research in this thesis, the results are interpreted as unequivocally positive for some of the CS variables selected, mainly from the control cluster and the cognitive triad but also touching upon emotional integration and interpersonal forcefulness. However, it is important to consider, that the results for other variables were consistently negative, and that the use of these variables in psychotherapy assessment undoubtedly is of questionable value.

\section{References}

Acklin, M. W. (1992). Psychodiagnosis of personality structure: Psychotic personality organisation. Journal of Personality Assessment, 58, 454-463.

Acklin, M. W., McDowell, C. J., Verschell, M. S., \& Chan, D. (2000). Interobserver agreement, intraobserver reliability, and the Rorschach Comprehensive System II. Journal of Personality Assessment, 74, 15-47.

Alpher, V.S., Perfetto, G.A., Henry, W.P., \& Strupp, H.H. (1990). The relationship between the Rorschach and assessment of the capacity to engage in shortterm dynamic psychotherapy. Psychotherapy, 27, 224-229.

American Psychiatric Association (1987). Diagnostic and statistical manual of mental disorders (3rd ed. Rev.). Washington, DC: Author.

American Psychiatric Association (1994). Diagnostic and statistical manual of mental disorders $\left(4^{\text {th }}\right.$ ed.). Washington, DC: Author.

Aronow, E. (2001). CS norms, psychometrics, and possibilities for the Rorshcach technique. Clinical Psychology: Science and Practice, 8, 383-385. 
Atkinson, L., Quarrington, B., Alp, I.E., \& Cyr, J.J. (1986). Rorschach validity: an empirical approach to the literature. Journal of Clinical Psychology, 42, 360362.

Baumann, B. D., Hilsenroth, M. J., Ackerman, S. J., Baity, M. R., Smith, C. L., Smith, S. R., et al. (2001). The Capacity for Dynamic Process Scale: An examination of reliability, validity, and relation to therapeutic alliance. Psychotherapy Research, 11, $275-294$.

Bihlar, B., \& Carlsson, A. M. (2000). An exploratory study of agreement between therapists' goals and patients' problems revealed by the Rorschach. Psychotherapy Research, 10, 196-214.

Bihlar, B., \& Carlsson, A. M. (2001). Planned and actual goals in psychodynamic psychotherapies: Do patients' personality characteristics relate to agreement? Psychotherapy Research, 11, 383-400.

Blais, M. A., Hilsenroth, M. J., Castlebury, F., Fowler, J. C., Baity, M. R. (2001). Predicting DSM-IV Cluster B personality disorder criteria from mmpi-2 and Rorschach data: a test of incremental validity. Journal of Personality Assessment, 76, 150-168.

Blatt, S. J., \& Ford, R. Q. (1994). Therapeutic change. An object relations perspective. New York and London: Plenum Press.

Bornstein, R. F. (1999). Criterion validity of objective and projective dependency tests: A meta-analytical assessment of behavioural prediction. Psychological Assessment, 11, 48-57.

Carlsson, A.M., Nygren, M., Clinton, D., \& Bihlar, B. (1996). The Stockholm Comparative Psychotherapy Study (COMPASS): Project presentation and preliminary Rorschach findings. Rorschachiana, 21, 30-46.

Carlsson, C. F., Kula, M. L., \& St. Laurent, C. M. (1997). Rorschach revised DEPI and CDI with inpatient major depressives and borderline personality disorder with major depression: Validity issues. Journal of clinical psychology, 53, $51-58$.

Cicchetti, D. V. (1994). Guidelines, criteria, and rules of thumb for evaluating normed and standardized assessment instruments in psychology. Psychological assessment, 6, 284-290.

Conte, H. R., Buckley, P., Picard, S., \& Karasu, B. (1995). Relationships between psychological mindedness and personality traits and ego functioning: Validity studies. Comprehensive Psychiatry, 153, 11 - 17.

Conte, H. R., Plutchik, R., Jung, B. B., Picard, S., Karasu, T. B., \& Lotterman, A. (1990). Psychological mindedness as a predictor of psychotherapy outcome: A preliminary report. Comprehensive Psychiatry, 31, 426 - 431.

Costello, R. M. (1999). Two foundations of Rorschach Assessment revisited. European Journal of Psychological Assessment, 15, 64-77.

Curran, P., West, S., \& Finch, J. (1996). The robustness of test statistics to nonnormality and specification error in confirmatory factor analysis. Psychological Methods, 1, 16-29.

Dies, R. R. (1995). Conceptual issues in Rorschach research. In J. E. Exner, Jr. (Ed.), 
The CS and assessment for dynamic psychotherapy

Issues and methods in Rorschach research (pp. 24-51). Hillsdale, NJ: Lawrence Erlbaum.

Exner, J. E., Jr. (1974). The Rorschach: A comprehensive system: Vol. 1 Basic foundations. New York: Wiley.

Exner, J. E., Jr. (1991). The Rorschach: A comprehensive system: Vol. 2. Interpretation (2nd ed.). New York: Wiley.

Exner, J. E., Jr. (1993). The Rorschach: A comprehensive system: Vol. 1 Basic foundations (3rd ed.). New York: Wiley.

Exner, J. E., Jr. (1995). A Rorschach workbook for the Comprehensive System ( $4^{\text {th }}$ ed.). Asheville, NC: Rorschach Workshops.

Exner, J. E., Jr. (1997). The future of Rorschach in personality assessment. Journal of Personality Assessment, 68, 37-46.

Exner, J. E., Jr. (2001). A comment on 'The misperception of psychopathology: problems with the norms of the Comprehensive system for the Rorschach'. Clinical Psychology: Science and Practice, 8, 386-388.

Exner, J. E., Jr. (2002). A new nonpatient sample for the Rorschach Comprehensive system. Journal of Personality Assessment, 78, 391-404.

Exner, J. E., Jr. (2003). The Rorschach: A comprehensive system: Vol. 1 Basic foundations (4th ed.). New York: Wiley.

Exner, J. E., Jr., \& Andrikof-Sanglade, A. (1992). Rorschach Changes Following Brief and Short-Term Therapy. Journal of Personality Assessment, 59, 59-71.

Ganellen, R. J. (2001). Weighing evidence for the Rorschach's validity: A response to Wood et al. (1999). Journal of Personality Assessment, 77, 1-15.

Garb, H. N. (2003). Observations on the validity of neuropsychological and personality assessment testing. Australian Psychologist, 38, 14-21.

Garb, H. N., Florio, C. M., \& Grove, W. M. (1998). The Validity of the Rorschach and the Minnesota Multiphasic Personality Inventory: Results from MetaAnalyses. Psychological Science, 9, 402-404.

Garb, H. N., Florio, C. M., \& Grove, W. M. (1999). The Rorscahch Controversery: Reply to Parker, Hunsley, and Hanson. Psychological Science, 10, 293-294.

Garb, H. N., Wood, J. M., Lilienfeld, S. O., \& Nezworski, M. T. (2002). Effective use of projective techniques an clinical practice: Let data help with the selection and interpretation. Professional Psychology: Research and Practice, 33, 454463.

Garb, H. N., Wood, J.M., Nezworski, M.T., Grove, W.M., \& Stejskal, W.J. (2001). Toward a Resolution of the Rorschach Controversy. Psychological Assessment, $13,433-448$.

Garfield, S. L. (1994). Research on client variables in psychotherapy. In A. E. Bergin, \& S. L. Garfield (Eds.), Handbook of psychotherapy and behavior change (4th ed.)(pp. 190-228). New York: Wiley.

Garfield, S. L. (2000). The Rorschach test in clinical diagnosis - a brief commentary. Journal of clinical psychology, 56, 431-434.

Hays, W. L., (1973). Statistics for the social sciences $\left(2^{\text {nd }}\right.$ edition). London, New York, Sydney and Toronto: Holt, Rinehart and Winston. 
Hibbard, S. (2003). A critique of Lilienfeld et al.'s (2000) 'The scientific status of projective techniques'. Journal of Personality Assessment, 80, 260-271.

Hiller, J. B., Rosenthal, R., Bornstein, R. F., Berry, D. T. R., \& Brunell-Neuleib, S. (1999). A Comparative Meta-Analyses of Rorschach and MMPI Validity. Psychological Assessment, 11, 278-296.

Hilsenroth, M.J., Fowler, C.J., Padawer, J.R., \& Handler, L. (1997). Narcissism in the Rorschach Revisited: Some Reflections on Empirical data. Psychological Assessment, 9, 113-121.

Hilsenroth, M. J., Handler, L., Toman, K. M., \& Padawer, J. R. (1995). Rorschach and MMPI-2 indices of early psychotherapy termination. Journal of Consulting and Clinical Psychology, 63, 956-965.

Høglend, P. (1993). Suitability for brief dynamic psychotherapy: Psychodynamic variables as predictors of outcome. Acta Psychiatrica Scandinavica, 88, 104- 110.

Høglend, P. (1996). Motivation for brief dynamic psychotherapy. Psychotherapy and Psychosomatics, 65, 209-215.

Høglend, P., Engelstad, V., Sørbye, O., Heyerdahl, O., \& Amlo, S. (1994). The role of insight in exploratory psychodynamic psychotherapy. British Journal of Medical Psychology, 67, 305-317.

Høglend, P., Sørbye, O., Sørlie, T., Fossum, A., \& Engelstad, V. (1992). Selection criteria for brief dynamic psychotherapy: Reliability, factor structure and longterm predictive validity. Psychotherapy and Psychosomatics, 57, 67-74.

Hunsley, J., \& Bailey, J.M. (1999). The Clinical Utility of the Rorschach: Unfulfilled Promises and an Uncertain Future. Psychological Assessment, 11, 266-277.

Hunsley, J., \& Bailey, J.M. (2001). Whither the Rorschach? An analysis of the Evidence. Psychological Assessment, 13, 472-485.

Hunsley, J., \& Di Giulio, G. (2001). Norms, norming and clinical assessment. Clinical Psychology: Science and Practice, 8, 378-382.

Janson, H., \& Olsson, U. (2001). A measure of agreement for interval or nominal multivariate observations. Educational and Psychological Measurement, 61, 277289.

Janson, H., \& Stattin, H. (2003). Prediction of adolescent and adult delinquency from childhood Rorschach ratings. Journal of Personality Assessment, 81, 5163.

Karon, B. P. (2000). The clinical interpretation of the Thematic Apperception Test, Rorschach, and other clinical data: A re-examination of statistical versus clinical prediction. Professional Psychology: Research and Practice, 31, 230-233.

Kernberg, O. F. (1975). Borderline conditions and pathological narcissism. New York: Aronson.

Keithly, L. J., Samples, S. J., \& Strupp, H. H. (1980). Patient motivation as a predictor of process outcome in psychotherapy. Psychotherapy and psychosomatics, 33, $87-97$.

Kleiger, J. H. (1992). A conceptual critique of the EA : es comparison in the Comprehensive Rorschach System. Psychological Assessment, 4, 288-296.

Kleiger, J. H. (1997). Rorschach shading responses: From a printer's error to 
The CS and assessment for dynamic psychotherapy

anintegrated psychoanalytical paradigm. Journal of Personality Assessment, 69, 342-365.

Klopfer, B., Ainsworth, M. D., Klopfer W. G., \& Holt, R. R. (1954). Developments in the Rorschach Technique. Vol. 1. Technique and Theory. Yonkers-on-Hudson, NY: World Book.

LaBarbera, J. D., \& Cornsweet, C. (1985). Rorschach predictors of therapeutic outcome in child psychiatric inpatient service. Journal of Personality Assessment, 49, 120-124.

Lerner, P. M. (1991). Psychoanalytic theory and the Rorschach. Hillsdale, NJ: Analytic Press.

Lilienfeld, S., O., Wood, J., M., \& Garb, H., N. (2000). The scientific Status of projective Techniques. Psychological Science In The Public Interest, 1, 27-66.

McCallum, M., \& Piper, W. E. (1990a). A psychological mindedness assessment procedure. Journal of consulting and clinical psychology, 2, 412-418.

McCallum, M., \& Piper, W. E. (1990b). A controlled study of effectiveness and patient suitability for short-term group psychotherapy. International Journal of Group Psychotherapy, 40, 431-452.

McCallum, M., Piper, W. E., \& Joyce, A. S. (1992). Dropping out from short-term group therapy. Psychotherapy, 29, 206-214.

McDowell, C., \& Acklin M. W. (1996). Standardizing procedures for calculating interrater reliability. Conceptual and empirical foundations. Journal of Personality Assessment, 66, 308-320.

McGrath, R.E. (2001). Toward more clinically relevant assessment research.Journal of Personality Assessment, 77, 307-332.

McGrath, R.E. (2003). Enhancing accuracy in observational test scoring: The Comprehensive System as a case example. Journal of Personality Assessment, 81, $104-110$.

McWilliams, N. (1994). Psychoanalytic Diagnosis. Understanding Personality Structure in the Clinical Process. New York: Guilford.

Meyer, G. J. (1992). The Rorschach's factor structure: A contemporary investigation and historical overview. Journal of Personality Assessment, 59, 117-136.

Meyer, G. J. (1997a). Assessing reliability: Critical corrections for a critical examination of the Rorschach Comprehensive System. Psychological Assessment, 9, 480489.

Meyer, G. J. (1997b). Thinking clearly about reliability: More critical corrections regarding the Rorschach Comprehensive System. Psychological Assessment, 9, 495-498.

Meyer, G. J. (2000). Incremental validity of the Rorschach Prognostic Scale over the MMPI Ego Strength and IQ. Journal of Personality Assessment, 74, 356-370.

Meyer, G.J. (2001). Evidence to correct misperceptions about Rorschach norms. Clinical Psychology: Science and Practice, 8, 389-396.

Meyer, G. J., \& Archer, J. P. (2001). The hard science of the Rorschach: What do we know and where do we go? Psychological assessment, 13, 486-502.

Meyer, G. J., \& Handler, L. (1997). Meta-Analysis of the Rorschach Prognostic Scale. 
Journal of Personality Assessment, 69, 1-38.

Meyer, G. J., \& Handler, L. (2000). Correction to Meyer and Handler (1997). Journal of Personality Assessment, 74, 504-506.

Meyer, G. J., Hilsenroth, M.J., Baxter, D., Exner, J. E., Jr., Fowler, J. C., Piers, C. C., \& Resnick, J. (2002). An examination of interrater reliability for scoring the Rorschach Comprehensive system in eight data sets. Journal of Personality Assessment, 78, 219-274.

Meyer, G. J., Viglioine, D. J., \& Exner, J. E., Jr. (2001). Superiority of Form\% over Lambda for research on the Rorschach. Journal of Personality Assessment, 76, $68-75$.

Moras, K., \& Strupp, H. H. (1982). Pretherapy interpersonal relations, patients' alliance and outcome in brief psychotherapy. Archives of General Psychiatry, 39, 405-409.

Nezworski, M.T., \& Wood, J. M. (1995). Narcissism in the Comprehensive System for the Rorschach. Clinical Psychology: Science and Practice, 2, 179-199.

Ogrodniczuk, J. S., Piper, W. E., Joyce, A. S., \& McCallum, M. (2001). Investigating follow-up outcome change using hierarchical linear modeling. Psychotherapy research, 11, 13-28.

Orlinsky, D. E., Grawe, K., \& Parks, B. (1994). Process and outcome in psychotherapy - noch einmal. In A. E. Bergin, \& S. L. Garfield (Eds.), Handbook of psychotherapy and behavior change (4th ed.) (pp. 190-228). New York: Wiley.

Parker, K. C. H., Hanson, R. K., \& Hunsley, J. (1988). MMPI, Rorschach, and WAIS: A meta-analytic comparison of reliability, stability, and validity. Psychological Bulletin, 103, 367-373.

Parker, K. C.H., Hunsley, J., \& Hanson, K. (1999). Old wine from old skins sometimes tastes like vinegar: A response to Garb, Florio and Grove. Psychological Science, 10, 291-292.

Piper, W. E., Azim, H. F. A., Joyce, A. S., McCallum, M., Nixon, G. W. H., \& Segal, P. S. (1991). Quality of object relations versus interpersonal functioning as predictors of therapeutic alliance and psychotherapy outcome. Journal of Nervous and Mental Disease, 179, 432-438.

Piper, W. E., Azim, H. F., McCallum, M., Joyce, A. S., \& Ogrodniczuk, J. S. (1999). Follow-up findings for interpretive and supportive forms of psychotherapy and patient personality variables. Journal of Consulting and Clinical Psychology, 67, 267-273.

Piper, W. E., \& Duncan, S. C. (1999). Object relations theory and short-term dynamic psychotherapy: Findings from the quality of object relations scale. Clinical psychology review, 19, 669-685.

Piper, W. E., Joyce, A. S., Azim, H. F. A., \& Rosie, J. S. (1994). Patient characteristics and success in day treatment. Journal of Nervous and Mental Disease, 182, 381-386.

Piper, W. E., Joyce, A. S., McCallum, M., \& Azim, H. F. (1998). Interpretive and supportive forms of psychotherapy and patient personality variables. Journal of Consulting and Clinical Psychology, 66, 558-567. 
The CS and assessment for dynamic psychotherapy

Piper, W. E., Joyce, A. S., Rosie, J. S., Azim, H. F. A. (1994). Psychological mindedness, work, and outcome in day treatment. International Journal of Group Therapy, 44, 291-311.

Rorschach, H. (1942). Psychodiagnostics: A diagnostic test based on perception. (P. Lemkau $\&$ B. Kroneberg, Trans.) Berne, Switzerland: Hans Huber. (Original work published 1921).

Rosenthal, R., Hiller, J. B., Bornstein, R. F., Berry, T. R., \& Brunell-Neulib, S. (2001). Meta-analytic methods, the Rorschach and the MMPI. Psychological Assessment, 13, 449-451.

Roth, A., \& Fonagy, P. (1996). What works for whom? A critical review of psychotherapy research. New York: Guilford Press.

Shaffer, T. W., Erdberg, P., \& Haroian, J. (1999). Current nonpatient data for the Rorschach, WAIS-R, and MMPI-2. Journal of Personality Assessment, 73, 305316.

Sifneos, P. E. (1978). Motivation for change: A prognostic guide for successful psychotherapy. Psychotherapy and Psychosomatics, 29, 293-298.

Tasca, G. A., Balfour, L., Bissada, H., Busby, K., Conrad, G., Cameron, P., et al. (1999). Treatment completion and outcome in a partial hospitalisation program: Interactions among patient variables. Psychotherapy Research, 9, 232 247.

Valbak, K. (2003). Suitability for psychoanalytic psychotherapy (Doctoral dissertation, University of Aarhus, 2003).

Viglione, D. J. (1995). Considerations regarding data analysis. In J. E. Exner, Jr. (Ed.), Issues and methods in Rorschach research (pp. 195-226). Hillsdale, NJ: Lawrence Erlbaum.

Viglione, D.J. (1997). Problems in Rorschach research and what to do about them. Journal of Personality Assessment, 68, 590-599.

Viglione, D.J. (1999). A review of Recent Research Addressing the Utility of the Rorschach. Psychological Assessment, 11, 251-265.

Viglione, D.J., \& Hilsenroth, J.M. (2001). The Rorschach: facts, fictions, and future. Psychological Assessment, 13, 452-471.

Weiner, I. B. (1996). Some observations on the validity of the Rorschach inkblot method. Psychological Assessment, 8, (2), 2066-213.

Weiner, I. B. (1998). Principles of Rorschach Interpretation. Hillsdale, NJ: Lawrence Erlbaum.

Weiner, I. B. (2000a). Making Rorschach interpretation as good as it can be. Journal of Personality Assessment, 74, 164-174.

Weiner, I. B. (2000b). Using The Rorschach properly in practice and research. Journal of Clinical Psychology, 56, 435-438.

Weiner, I. B. (2001). Advancing the Science of Psychological Assessment: The Rorschach Inkblot Method as Exemplar. Psychological Assessment, 13, 423-432.

Weiner, I.B., \& Exner, J.E., Jr. (1991). Rorschach changes in long-term and shortterm psychotherapy. Journal of Personality Assessment, 56, 453-465.

Wood, J. M., Lilienfield, S.O., Garb, H.N., \& Nezworski, M.T. (2000a). The Ror- 
schach Test in Clinical Diagnosis: A Critical Review, with a Backward Look at Garfield (1947). Journal of Clinical Psychology, 56, 395-430.

Wood, J. M., Lilienfield, S.O., Garb, H.N., \& Nezworski, M.T. (2000b). Limitations of the Rorschach as a diagnostic tool: A reply to Garfield (2000), Lerner (2000) and Weiner (2000). Journal of Clinical Psychology, 56, 441-448.

Wood, J.M., Lilienfeld, S.O., Nezworski, M.T., \& Garb, H. N. (2001). Coming to grips with negative evidence for the Comprehensive System for the Rorschach: A comment on Gacono, Loving, and Bodholt; Ganellen; and Bornstein. Journal of Personality Assessment, 77, 48-70.

Wood, J. M., Nezworski, M.T., Garb, H.N., \& Lilienfield, S.O. (2001a). The misperception of psychopathology: Problems with the norms of the Comprehensive system for the Rorschach. Clinical Psychology: Science and Practice, 8, 350373.

Wood, J. M., Nezworski M. T., Garb, H. N., \& Lilienfield, S. O. (2001b). problems with the norms of the Comprehensive System for the Rorschach: Methodological and conceptual considerations. Clinical Psychology: Science and Practice, 8, 397-402.

Wood, J. M., Nezworski, M. T., \& Stejskal, W. J. (1996a). The Comprehensive System for the Rorschach: A critical examination. Psychological Science, 7, 3-10.

Wood, J. M., Nezworski, M. T., \& Stejskal, W. J. (1996b). Thinking critically about the Comprehensive System for the Rorschach: A reply to Exner. Psychological Science, 7, 14-17.

Wood, J. M., Nezworski, M. T., \& Stejskal, W. J. (1997). The reliability of the Comprehensive System for the Rorschach: A comment on Meyer (1997). Psychological Assessment, 9, 490-494. 
